\title{
CANCELLATION THEOREM FOR FRAMED MOTIVES OF ALGEBRAIC VARIETIES
}

\author{
A. ANANYEVSKIY, G. GARKUSHA, AND I. PANIN
}

\begin{abstract}
The machinery of framed (pre)sheaves was developed by Voevodsky [V1]. Based on the theory, framed motives of algebraic varieties are introduced and studied in [GP1]. An analog of Voevodsky's Cancellation Theorem [V2] is proved in this paper for framed motives stating that a natural map of framed $S^{1}$-spectra

$$
M_{f r}(X)(n) \rightarrow \underline{\operatorname{Hom}}\left(\mathbb{G}, M_{f r}(X)(n+1)\right), \quad n \geqslant 0,
$$

is a schemewise stable equivalence, where $M_{f r}(X)(n)$ is the $n$th twisted framed motive of $X$. This result is also necessary for the proof of the main theorem of [GP1] computing fibrant resolutions of suspension $\mathbb{P}^{1}$-spectra $\Sigma_{\mathbb{P 1}}^{\infty} X_{+}$with $X$ a smooth algebraic variety.

The Cancellation Theorem for framed motives is reduced to the Cancellation Theorem for linear framed motives stating that the natural map of complexes of abelian groups
\end{abstract}

$$
\mathbb{Z} \mathrm{F}\left(\Delta^{\bullet} \times X, Y\right) \rightarrow \mathbb{Z} \mathrm{F}\left(\left(\Delta^{\bullet} \times X\right) \wedge\left(\mathbb{G}_{m}, 1\right), Y \wedge\left(\mathbb{G}_{m}, 1\right)\right), \quad X, Y \in S m / k,
$$

is a quasi-isomorphism, where $\mathbb{Z F}(X, Y)$ is the group of stable linear framed correspondences in the sense of [GP1].

\section{INTRODUCTION}

The main goal of the Voevodsky theory on framed correspondences (see [V1, Introduction]) is to suggest a new approach to the stable motivic homotopy theory $S H(k)$ over a field $k$. This approach is more amenable to explicit calculations. Recall that Voevodsky [V1, Section 2] invented a category of framed correspondences $F r_{*}(k)$ whose objects are those of $S \mathrm{~m} / k$ and morphisms sets $F r_{*}(X, Y)=\sqcup_{n \geqslant 0} F r_{n}(X, Y)$ are defined by means of certain geometric data. The elements of $F_{n}(X, Y)$ are called framed correspondences of level $n$. The definition of $F r_{*}(k)$ is recalled in Section 2 below. For every $Y \in S m / k$ there is a distinguished morphism $\sigma_{Y}=(Y \times 0, Y \times$ $\left.\mathbb{A}^{1}, t, p r_{Y}\right) \in F_{1}(Y, Y)$. Following Voevodsky [V1], we denote by

$$
F r(X, Y):=\operatorname{colim}\left(F r_{0}(X, Y) \stackrel{\sigma_{Y}}{\longrightarrow} F r_{1}(X, Y) \stackrel{\sigma_{Y}}{\longrightarrow} \ldots \stackrel{\sigma_{Y}}{\longrightarrow} F r_{n}(X, Y) \stackrel{\sigma_{Y}}{\longrightarrow} \ldots\right)
$$

and refer to it as the set of stable framed correspondences. Replacing $Y$ by a simplicial object $Y^{\bullet}$ in $S m / k$, we get a simplicial set $\operatorname{Fr}\left(X, Y^{\bullet}\right)$. Finally, one can take the diagonal of the pointed bisimplicial set $\operatorname{Fr}\left(\Delta^{\bullet} \times X, Y^{\bullet}\right)$. Voevodsky conjectured that if the motivic space $\operatorname{Fr}\left(\Delta^{\bullet} \times-, Y^{\bullet}\right)$ is locally connected in the Nisnevich topology, then it is isomorphic in $H_{\mathbb{A}^{1}}(k)$ to the motivic space $\Omega_{\mathbb{P}^{1}}^{\infty} \Sigma_{\mathbb{P}^{1}}^{\infty}\left(Y_{+}^{\bullet}\right)$. In particular, the theory of framed correspondences gives a machinery for computing motivic infinite loop spaces.

Inspired by the Voevodsky theory [V1], the theory of framed motives of algebraic varieties is introduced and developed in [GP1]. As an application, the above Voevodsky conjecture is solved in [GP1, Section 10] in the affirmative. Moreover, under the above assumption on $Y^{\bullet}$ the motivic space $\operatorname{Fr}\left(\Delta^{\bullet} \times-, Y^{\bullet}\right)$ is $\mathbb{A}^{1}$-local. This result can be regarded as a motivic counterpart of the Segal

2010 Mathematics Subject Classification. 14F42, 14F05.

Key words and phrases. Motivic homotopy theory, framed motives, cancellation theorem. 
theorem. Also, an alternative approach to the classical Morel-Voevodsky [MV] stable homotopy theory $S H(k)$ is suggested in [GP3], which is based on the machinery of framed bispectra. One of the key steps in the computations of [GP1, GP3] is Theorem A proved in this paper. Theorem A is the main result of the present paper. In order to state it, we have to recall some definitions and constructions from [GP1].

The framed motive of $X \in S m / k$ is an explicitly constructed $S^{1}$-spectrum $M_{f r}(X)$, which is connected and an $\Omega$-spectrum in positive degrees (see [GP1] for details). Following the notation of [GP1, Section 8] let $\mathbb{G}$ be the cone $\left(\mathbb{G}_{m}\right)_{+} / / p t_{+}$of the embedding $p t_{+} \stackrel{1}{\hookrightarrow}\left(\mathbb{G}_{m}\right)_{+}$in the category of pointed simplicial presheaves $\operatorname{sPre}_{.}(\mathrm{Sm} / \mathrm{k})$. Its sheafification is represented in the category $\Delta^{\mathrm{op}}\left(F_{0}(k)\right)$ by the object $\mathbb{G}_{m}^{\wedge 1}$ (see [GP1, Notation 8.1]). For any integer $n \geqslant 1$ let $\mathbb{G}_{m}^{\wedge n}$ be the $n$th monoidal power of $\mathbb{G}_{m}^{\wedge 1}$ in the symmetric monoidal category $\Delta^{\mathrm{op}}\left(F r_{0}(k)\right)$ (see [GP1, Notation 8.1]). For a variety $X \in S m / k$ let $M_{f r}\left(X \times \mathbb{G}_{m}^{\wedge n}\right)$ be the framed motive of the simplicial object $X \times \mathbb{G}_{m}^{\wedge n} \in \Delta^{\mathrm{op}}\left(F r_{0}(k)\right)$. It is an explicitly constructed $S^{1}$-spectrum which is connected and an $\Omega$ spectrum in positive degrees (see [GP1, Sections 5 and 6] for details). For brevity we also write $M_{f r}(X)(n)$ to denote $M_{f r}\left(X \times \mathbb{G}_{m}^{\wedge n}\right)$ and call $M_{f r}(X)(n)$ the $n$-twisted framed motive of $X$ (see [GP1, Section 11]). The main object of [GP1] is the bispectrum

$$
M_{f r}^{\mathbb{G}}(X)=\left(M_{f r}(X), M_{f r}(X)(1), M_{f r}(X)(2), \ldots\right),
$$

each term of which is a twisted framed motive of $X$ and structure maps of the bispectra

$$
M_{f r}(X)(n) \rightarrow \underline{\operatorname{Hom}}\left(\mathbb{G}, M_{f r}(X)(n+1)\right),
$$

are defined in [GP1, Section 11] (they use [GP1, "General Framework" of Section 5]).

The major property of the bispectrum $M_{f r}^{\mathbb{G}}(X)$ is that its levelwise Nisnevich local stable replacement $M_{f r}^{\mathbb{G}}(X)_{f}$ is a fibrant replacement of the suspension bispectrum $\Sigma_{\mathbb{G}^{\infty}}^{\infty} \Sigma_{S^{1}}^{\infty} X_{+}$. This may also be viewed as a motivic version of the Barratt, Priddy, and Quillen theorem. The proof of this major property is given in [GP1] and is heavily based on the Cancellation Theorem.

The main purpose of the paper is to prove the following (cf. Voevodsky [V2])

Theorem A (Cancellation). Let $k$ be an infinite perfect field, $X \in S m / k$ and $n \geqslant 0$. Then the following statements are true:

(1) the natural map of $S^{1}$-spectra

$$
M_{f r}(X)(n) \rightarrow \underline{\operatorname{Hom}}\left(\mathbb{G}, M_{f r}(X)(n+1)\right)
$$

is a schemewise stable equivalence;

(2) the induced map of $S^{1}$-spectra

$$
M_{f r}(X)(n)_{f} \rightarrow \underline{\operatorname{Hom}}\left(\mathbb{G}, M_{f r}(X)(n+1)_{f}\right)
$$

is a schemewise stable equivalence. Here $M_{f_{r}}(X)(n)_{f}$ and $M_{f_{r}}(X)(n+1)_{f}$ are Nisnevich local stable fibrant replacements of $M_{f r}(X)(n)$ and $M_{f r}(X)(n+1)$ in the injective local stable model structure of $S^{1}$-spectra.

As an application of Theorem A we prove the following

Theorem B. Let $k$ be an infinite perfect field, $X \in S m / k$ and $n \geqslant 0$. Then the bispectrum

$$
M_{f r}^{\mathbb{G}}(X)_{f}=\left(M_{f r}(X)_{f}, M_{f r}(X)(1)_{f}, M_{f r}(X)(2)_{f}, \ldots\right)
$$

obtained from $M_{f r}^{\mathbb{G}}(X)$ by taking levelwise Nisnevich local stable fibrant replacements with structure maps those of Theorem A(2) is a motivically fibrant $\left(S^{1}, \mathbb{G}\right)$-bispectrum. 
The main strategy of proving Theorem A is to reduce it to the "Linear Cancellation Theorem". In order to formulate it, recall from [GP1, Definition 8.3] that the category $\mathbb{Z} \mathrm{F}_{*}(k)$ is the additive category whose objects are those of $S m / k$ with Hom-groups described in Definition 2.4. Briefly speaking, for every $n \geqslant 0$ and $X, Y \in S m / k$ we set

$$
\mathbb{Z} \mathrm{F}_{n}(X, Y):=\mathbb{Z} \mathrm{Fr}_{n}(X, Y) /\left\langle Z_{1} \sqcup Z_{2}-Z_{1}-Z_{2}\right\rangle,
$$

where $Z_{1}, Z_{2}$ are supports of framed correspondences level $n$ in the sense of Voevodsky [V1] (see Definition 2.4 as well). In other words, $\mathbb{Z F}_{n}(X, Y)$ is the free abelian group generated by the framed correspondences of level $n$ with connected supports. We then set

$$
\operatorname{Hom}_{\mathbb{Z} \mathrm{F}_{*}(k)}(X, Y):=\bigoplus_{n \geqslant 0} \mathbb{Z F}_{n}(X, Y) .
$$

Given smooth varieties $X, Y \in S m / k$ and $n \geqslant 0$, there is a canonical suspension morphism $\Sigma: \mathbb{Z F}_{n}(X, Y) \rightarrow \mathbb{Z F}_{n+1}(X, Y)$. We can stabilise in the $\Sigma$-direction to get an abelian group (see Definition 2.6)

$$
\mathbb{Z} \mathrm{F}(X, Y):=\operatorname{colim}\left(\mathbb{Z F}_{0}(X, Y) \stackrel{\Sigma}{\rightarrow} \mathbb{Z}_{1}(X, Y) \stackrel{\Sigma}{\rightarrow} \cdots\right) .
$$

The presheaf $\mathbb{Z} \mathrm{F}(Y):=\mathbb{Z} \mathrm{F}(-, Y)$ has a canonical structure of a $\mathbb{Z F} \mathrm{F}_{*}(k)$-presheaf. For each scheme $Y \in S m / k$ and each scheme $S \in S m / k$ pointed at a $k$-rational point $s \in S$, the natural functor

$$
\nabla: \operatorname{Pre}_{A b}\left(\mathbb{Z F}_{*}(k)\right) \times \operatorname{Pre}_{A b}\left(\mathbb{Z F}_{0}(k)\right) \rightarrow \operatorname{Pre}_{A b}\left(\mathbb{Z F}_{*}(k)\right)
$$

defined on p. 6 takes the pair $(\mathbb{Z F}(Y),(S, s))$ to the $\mathbb{Z} \mathrm{F}_{*}(k)$-presheaf $\mathbb{Z F}(Y) \otimes(S, s)$ which we also denote by $\mathbb{Z} \mathrm{F}(Y \wedge(S, s))$. By the General Framework of [GP1, Section 5] (also see p. 6) one has a $\mathbb{Z} \mathrm{F}_{*}(k)$-presheaf $\underline{\operatorname{Hom}}((S, s), \mathbb{Z F}(Y \wedge(S, s)))$ together with a morphism of $\mathbb{Z} \mathrm{F}_{*}(k)$-presheaves

$$
\mathbb{Z} \mathrm{F}(Y) \stackrel{-\otimes(S, s)}{\longrightarrow} \underline{\operatorname{Hom}}((S, s), \mathbb{Z} \mathrm{F}(Y \wedge(S, s)) .
$$

The Linear Cancellation Theorem is formulated as follows (see Section 2 for details).

Theorem $\mathbf{C}$ (Linear Cancellation). Let $k$ be an infinite perfect field and let $Y$ be a $k$-smooth scheme. Then

$$
-\otimes\left(\mathbb{G}_{m}, 1\right): \mathbb{Z} \mathrm{F}\left(\Delta^{\bullet} \times-, Y\right) \rightarrow \underline{\operatorname{Hom}}\left(\left(\mathbb{G}_{m}, 1\right), \mathbb{Z F}\left(\Delta^{\bullet} \times-, Y \wedge\left(\mathbb{G}_{m}, 1\right)\right)\right.
$$

is a quasi-isomorphism of complexes of $\mathbb{Z} \mathrm{F}_{*}(k)$-presheaves of abelian groups. Here $\Delta^{\bullet}$ is the standard cosimplicial object in $\mathrm{Sm} / k$.

One of the main computational results of [GNP] says that schemewise homology of the complex $\mathbb{Z} \mathrm{F}\left(\Delta^{\bullet} \times-, Y\right)$ computes homology of the framed motive $M_{f r}(Y)$ of $Y \in S m / k$. Moreover, the complex represents the "linear framed motive" of $Y$ (see [GNP] for details).

Throughout the paper the base field $k$ is supposed to be infinite. We also employ the following notation:

- all schemes are separated Noetherian $k$-schemes, all morphisms of schemes are $k$ morphisms; write pt for the scheme $\operatorname{Spec}(k)$.

- $S m / k$ is the category of smooth $k$-schemes of finite type;

- we refer to the objects of $S m / k$ as $k$-smooth schemes or smooth $k$-schemes;

- Following [GrD], by an essentially smooth $k$-scheme we mean a Noetherian $k$-scheme $X$ which is the inverse limit of a left filtering system $\left(X_{i}\right)_{i \in I}$ with each transition morphism $X_{i} \rightarrow X_{j}$ being an étale affine morphism between smooth $k$-schemes. 


\section{PRELIMINARIES}

In this section we collect basic facts for framed correspondences. We start with preparations.

Let $V$ be a scheme and $Z$ be a closed subscheme. Recall that an étale neighborhood of $Z$ in $V$ is a triple $\left(W^{\prime}, \pi^{\prime}: W^{\prime} \rightarrow V, s^{\prime}: Z \rightarrow W^{\prime}\right)$ satisfying the following conditions:

(i) $\pi^{\prime}$ is an étale morphism;

(ii) $\pi^{\prime} \circ s^{\prime}$ coincides with the inclusion $Z \hookrightarrow V$ (thus $s^{\prime}$ is a closed embedding);

(iii) $\left(\pi^{\prime}\right)^{-1}(Z)=s^{\prime}(Z)$.

A morphism between two étale neighborhoods $\left(W^{\prime}, \pi^{\prime}, s^{\prime}\right) \rightarrow\left(W^{\prime \prime}, \pi^{\prime \prime}, s^{\prime \prime}\right)$ of $Z$ in $V$ is a morphism $\rho: W^{\prime} \rightarrow W^{\prime \prime}$ such that $\pi^{\prime \prime} \circ \rho=\pi^{\prime}$ and $\rho \circ s^{\prime}=s^{\prime \prime}$. Note that such $\rho$ is automatically étale by [EGA4, VI.4.7].

Definition 2.1 (Voevodsky [V1]). For $k$-smooth schemes $X, Y$ and $n \geqslant 0$ an explicit framed correspondence $\Phi$ of level $n$ consists of the following data:

(1) a closed subset $Z$ in $\mathbb{A}_{X}^{n}$ which is finite over $X$;

(2) an etale neighborhood $p: U \rightarrow \mathbb{A}_{X}^{n}$ of $Z$ in $\mathbb{A}_{X}^{n}$;

(3) a collection of regular functions $\varphi=\left(\varphi_{1}, \ldots, \varphi_{n}\right)$ on $U$ such that $\cap_{i=1}^{n}\left\{\varphi_{i}=0\right\}=Z$;

(4) a morphism $g: U \rightarrow Y$.

The subset $Z$ will be referred to as the support of the correspondence. We shall also write triples $\Phi=(U, \varphi, g)$ or quadruples $\Phi=(Z, U, \varphi, g)$ to denote explicit framed correspondences.

Two explicit framed correspondences $\Phi$ and $\Phi^{\prime}$ of level $n$ are said to be equivalent if they have the same support and there exists an etale neighborhood $V$ of $Z$ in $U \times_{\mathbb{A}_{X}^{n}} U^{\prime}$ such that the morphism $g \circ p r$ agrees with $g^{\prime} \circ p r^{\prime}$ and $\varphi \circ p r$ agrees with $\varphi^{\prime} \circ p r^{\prime}$ on $V$. A framed correspondence of level $n$ is an equivalence class of explicit framed correspondences of level $n$.

We let $\operatorname{Fr}_{n}(X, Y)$ denote the set of framed correspondences from $X$ to $Y$. It as a pointed set with the distinguished point being the class $0_{n}$ of the explicit correspondence with $U=\emptyset$.

As an example, the set $\operatorname{Fr}_{0}(X, Y)$ coincides with the set of pointed morphisms $X_{+} \rightarrow Y_{+}$. In particular, for a connected scheme $X$ one has

$$
\operatorname{Fr}_{0}(X, Y)=\operatorname{Hom}_{S m / k}(X, Y) \sqcup\left\{0_{0}\right\} .
$$

If $f: X^{\prime} \rightarrow X$ is a morphism of schemes and $\Phi=(U, \varphi, g)$ an explicit correspondence from $X$ to $Y$ then

is an explicit correspondence from $X^{\prime}$ to $Y$.

$$
f^{*}(\Phi):=\left(U^{\prime}=U \times_{X} X^{\prime}, \varphi \circ p r, g \circ p r\right)
$$

The following definition is to describe compositions of framed correspondences.

Definition 2.2. Let $X, Y$ and $S$ be $k$-smooth schemes and let $a=\left(Z, U,\left(\varphi_{1}, \varphi_{2}, \ldots, \varphi_{n}\right), g\right)$ be an explicit correspondence of level $n$ from $X$ to $Y$ and let $b=\left(Z^{\prime}, U^{\prime},\left(\psi_{1}, \psi_{2}, \ldots, \psi_{m}\right), g^{\prime}\right)$ be an explicit correspondence of level $m$ from $Y$ to $S$. We define their composition as an explicit correspondence of level $n+m$ from $X$ to $S$ by

$$
\left(Z \times_{Y} Z^{\prime}, U \times_{Y} U^{\prime},\left(\varphi_{1}, \varphi_{2}, \ldots, \varphi_{n}, \psi_{1}, \psi_{2}, \ldots, \psi_{m}\right), g^{\prime}\right) .
$$

Clearly, the composition of explicit correspondences respects the equivalence relation on them and defines associative pairings

$$
\operatorname{Fr}_{n}(X, Y) \times \operatorname{Fr}_{m}(Y, S) \rightarrow \operatorname{Fr}_{n+m}(X, S) .
$$

Given $X, Y \in S m / k$, denote by $\operatorname{Fr}_{*}(X, Y)$ the set $\bigsqcup_{n} \operatorname{Fr}_{n}(X, Y)$. The composition of framed correspondences defined above gives a category $\mathrm{Fr}_{*}(k)$. Its objects are those of $S m / k$ and the morphisms 
are given by the sets $\mathrm{Fr}_{*}(X, Y), X, Y \in S m / k$. Since the naive morphisms of schemes can be identified with certain framed correspondences of level zero, we get a canonical functor

$$
\mathrm{Sm} / k \rightarrow \mathrm{Fr}_{*}(k) \text {. }
$$

One can easily see that for a framed correspondence $\Phi: X \rightarrow Y$ and a morphism $f: X^{\prime} \rightarrow X$, one has $f^{*}(\Phi)=\Phi \circ f$.

Definition 2.3. Let $X, Y, S$ and $T$ be smooth schemes. There is an external product

$$
\operatorname{Fr}_{n}(X, Y) \times \operatorname{Fr}_{m}(S, T) \stackrel{-\bigotimes-}{\longrightarrow} \operatorname{Fr}_{n+m}(X \times S, Y \times T)
$$

given by

$$
\begin{gathered}
\left(Z, U,\left(\varphi_{1}, \varphi_{2}, \ldots, \varphi_{n}\right), g\right) \otimes\left(Z^{\prime}, U^{\prime},\left(\psi_{1}, \psi_{2}, \ldots, \psi_{m}\right), g^{\prime}\right)= \\
\left(Z \times Z^{\prime}, U \times U^{\prime},\left(\varphi_{1}, \varphi_{2}, \ldots, \varphi_{n}, \psi_{1}, \psi_{2}, \ldots, \psi_{m}\right), g \times g^{\prime}\right) .
\end{gathered}
$$

For the constant morphism $c: \mathbb{A}^{1} \rightarrow \mathrm{pt}$, we set (following Voevodsky [V1])

$$
\Sigma=-\otimes\left(t, c,\{0\}, \mathbb{A}^{1}, t, c\right): \operatorname{Fr}_{n}(X, Y) \rightarrow \mathrm{Fr}_{n+1}(X, Y)
$$

and refer to it as the suspension. If there is no likelihood of confusion, we shall also write $\Sigma$ to denote the element $1 \cdot\left(t, c,\{0\}, \mathbb{A}^{1}, t, c\right)$ in $\mathbb{Z} \mathrm{F}_{1}(\mathrm{pt}, \mathrm{pt})$ and $\Sigma^{n}$ for $\Sigma \otimes \ldots \otimes \Sigma$ in $\mathbb{Z} \mathrm{F}_{n}(\mathrm{pt}, \mathrm{pt})$. It will always be clear from the context which of the meanings for $\Sigma$ is used (either as the suspension or as the element in $\mathbb{Z} \mathrm{F}_{1}(\mathrm{pt}, \mathrm{pt})$ ).

Also, following Voevodsky [V1], one puts

$$
\operatorname{Fr}(X, Y)=\operatorname{colim}\left(\operatorname{Fr}_{0}(X, Y) \stackrel{\Sigma}{\rightarrow} \operatorname{Fr}_{1}(X, Y) \stackrel{\Sigma}{\rightarrow} \ldots \stackrel{\Sigma}{\rightarrow} \operatorname{Fr}_{n}(X, Y) \stackrel{\Sigma}{\rightarrow} \ldots\right)
$$

and refer to it as the set of stable framed correspondences. The above external product induces external products

$$
\begin{aligned}
& \operatorname{Fr}_{n}(X, Y) \times \operatorname{Fr}(S, T) \stackrel{-\unrhd-}{\longrightarrow} \operatorname{Fr}(X \times S, Y \times T), \\
& \operatorname{Fr}(X, Y) \times \operatorname{Fr}_{0}(S, T) \stackrel{-\unrhd-}{\longrightarrow} \operatorname{Fr}(X \times S, Y \times T) .
\end{aligned}
$$

Recall now the definition of the category of linear framed correspondences $\mathbb{Z} \mathrm{F}_{*}(k)$.

Definition 2.4. (see [GP1]) Let $X$ and $Y$ be smooth schemes. Denote by

$\diamond \mathbb{Z} \operatorname{Fr}_{n}(X, Y):=\widetilde{\mathbb{Z}}\left[\operatorname{Fr}_{n}(X, Y)\right]=\mathbb{Z}\left[\operatorname{Fr}_{n}(X, Y)\right] / \mathbb{Z} \cdot 0_{n}$, i.e the free abelian group generated by the set $\mathrm{Fr}_{n}(X, Y)$ modulo $\mathbb{Z} \cdot 0_{n}$;

$\diamond \mathbb{Z F}_{n}(X, Y):=\mathbb{Z F r}_{n}(X, Y) / A$, where $A$ is a subgroup generated by the elementts

$$
\begin{aligned}
& \left(Z \sqcup Z^{\prime}, U,\left(\varphi_{1}, \varphi_{2}, \ldots, \varphi_{n}\right), g\right)- \\
& \quad-\left(Z, U \backslash Z^{\prime},\left.\left(\varphi_{1}, \varphi_{2}, \ldots, \varphi_{n}\right)\right|_{U \backslash Z^{\prime}},\left.g\right|_{U \backslash Z^{\prime}}\right)-\left(Z^{\prime}, U \backslash Z,\left.\left(\varphi_{1}, \varphi_{2}, \ldots, \varphi_{n}\right)\right|_{U \backslash Z},\left.g\right|_{U \backslash Z}\right) .
\end{aligned}
$$

We shall also refer to the latter relation as the additivity property for supports. In other words, it says that a framed correspondence in $\mathbb{Z F}_{n}(X, Y)$ whose support is a disjoint union $Z \sqcup Z^{\prime}$ equals the sum of the framed correspondences with supports $Z$ and $Z^{\prime}$ respectively. Note that $\mathbb{Z}_{n}(X, Y)$ is $\mathbb{Z}\left[\operatorname{Fr}_{n}(X, Y)\right]$ modulo the subgroup generated by the elements as above, because $0_{n}=0_{n}+0_{n}$ in this quotient group, hence $0_{n}$ equals zero. Indeed, it is enough to observe that the support of $0_{n}$ equals $\emptyset \sqcup \emptyset$ and then apply the above relation to this support.

The elements of $\mathbb{Z} \mathrm{F}_{n}(X, Y)$ are called linear framed correspondences of level $n$ or just linear framed correspondences. 
Denote by $\mathbb{Z F}_{*}(k)$ the additive category whose objects are those of $S m / k$ with Hom-groups defined as

$$
\operatorname{Hom}_{\mathbb{Z} \mathrm{F}_{*}(k)}(X, Y)=\bigoplus_{n \geqslant 0} \mathbb{Z} \mathrm{F}_{n}(X, Y) .
$$

The composition is induced by the composition in the category $\mathrm{Fr}_{*}(k)$. Denote by $\operatorname{Pre}_{A b}\left(\mathbb{Z F}_{*}(k)\right)$ the Grothendieck category of additive presheaves of abelian groups on the additive category $\mathbb{Z} \mathrm{F}_{*}(k)$.

Denote by $\mathbb{Z F}_{0}(k)$ the additive category whose objects are those of $S m / k$ with Hom-groups defined as $\operatorname{Hom}_{\mathbb{Z} \mathrm{F}_{0}(k)}(X, Y)=\mathbb{Z F}_{0}(X, Y)$. Clearly, $\mathbb{Z} \mathrm{F}_{0}(k)$ is an additive subcategory of the additive category $\mathbb{Z} \mathrm{F}_{*}(k)$. Finally, denote by $\operatorname{Pre}_{A b}\left(\mathbb{Z F}_{0}(k)\right)$ the category of additive presheaves of abelian groups on the additive category $\mathbb{Z F}_{0}(k)$.

There is a natural functor from $S m / k$ to $\mathbb{Z} \mathrm{F}_{0}(k)$. It is the identity on objects and takes a regular morphism $f: X \rightarrow Y$ to the linear framed correspondence $1 \cdot\left(X, X \times \mathbb{A}^{0}, \operatorname{pr}_{\mathbb{A}^{0}}, f \circ p r_{X}\right) \in \mathbb{Z F}_{0}(k)$.

Definition 2.5. Let $X, Y, S$ and $T$ be schemes. The external product from Definition 2.3 induces a unique external product

$$
\mathbb{Z} \mathrm{F}_{n}(X, Y) \times \mathbb{Z} \mathrm{F}_{m}(S, T) \stackrel{-\otimes-}{\longrightarrow} \mathbb{Z} \mathrm{F}_{n+m}(X \times S, Y \times T)
$$

such that for any elements $a \in \operatorname{Fr}_{n}(X, Y)$ and $b \in \operatorname{Fr}_{m}(S, T)$ one has $1 \cdot a \otimes 1 \cdot b=1 \cdot(a \otimes b) \in$ $\mathbb{Z} \mathrm{F}_{n+m}(X \times S, Y \times T)$.

Definition 2.6. For any $k$-smooth variety $Y$, the presheaf represented by $Y$ is denoted by $\mathbb{Z} \mathrm{F}_{*}(-, Y)$. One of the main $\mathbb{Z} \mathrm{F}_{*}(k)$-presheaves of this paper is defined as

$$
\mathbb{Z} \mathrm{F}(-, Y)=\operatorname{colim}\left(\mathbb{Z}_{0}(-, Y) \stackrel{\Sigma}{\rightarrow} \mathbb{Z}_{1}(-, Y) \stackrel{\Sigma}{\rightarrow} \ldots \stackrel{\Sigma}{\rightarrow} \mathbb{Z}_{n}(-, Y) \stackrel{\Sigma}{\rightarrow} \ldots\right) .
$$

For a $k$-smooth variety $X$, the elements of $\mathbb{Z} \mathrm{F}(X, Y)$ are also called stable linear framed correspondences. Notice that stable linear framed correspondences do not form morphisms of a category.

General Framework. The pairing $\otimes$ of Definition 2.5 gives rise to a functor

$$
\mathbb{Z} \mathrm{F}_{*}(k) \times \mathbb{Z} \mathrm{F}_{0}(k) \stackrel{\otimes}{\rightarrow} \mathbb{Z} \mathrm{F}_{*}(k)
$$

taking a pair of schemes $(X, S)$ to $X \times S$ and taking a pair of morphisms $(a, b)$ to the morphism $a \otimes b$. It is naturally extended to a functor

$$
\operatorname{Pre}_{A b}\left(\mathbb{Z F}_{*}(k)\right) \times \operatorname{Pre}_{A b}\left(\mathbb{Z F}_{0}(k)\right) \stackrel{\otimes}{\rightarrow} \operatorname{Pre}_{A b}\left(\mathbb{Z F}_{*}(k)\right) .
$$

Given schemes $Y, S \in \mathbb{Z F}_{0}(k)$, consider the presheaf $\mathbb{Z F}(S)$ in $\operatorname{Pre}_{A b}\left(\mathbb{Z F}_{0}(k)\right)$ and the presheaf $\mathbb{Z F}(Y)$ in $\operatorname{Pre}_{A b}\left(\mathbb{Z F}_{*}(k)\right)$. Similarly to [GP1, General Framework, Section 5] there are defined $\mathbb{Z} \mathrm{F}_{*}(k)$-presheaves $\mathbb{Z} \mathrm{F}(Y) \otimes S$ and $\underline{\operatorname{Hom}}(S, \mathbb{Z} \mathrm{F}(Y) \otimes S)$ as well as a natural $\mathbb{Z} \mathrm{F}_{*}(k)$-morphism $\mathbb{Z} \mathrm{F}(Y) \stackrel{-\otimes S}{\longrightarrow} \underline{\operatorname{Hom}}(S, \mathbb{Z} \mathrm{F}(Y) \otimes S)$. By construction, $\mathbb{Z} \mathrm{F}(Y) \otimes S=\mathbb{Z} \mathrm{F}(Y \times S)$. Thus one has the following morphism of $\mathbb{Z} \mathrm{F}_{*}(k)$-presheaves

$$
-\otimes i d_{S}: \mathbb{Z} \mathrm{F}(Y) \rightarrow \underline{\operatorname{Hom}}(S, \mathbb{Z F}(Y \times S))
$$

taking $a \in \mathbb{Z} \mathrm{F}(X, Y)$ to $a \otimes i d_{S} \in \mathbb{Z} \mathrm{F}(X \times S, Y \times S)$.

Definition 2.7. Let $(S, s)$ be a $k$-smooth pointed scheme. Then the morphism $e_{S}: S \rightarrow \mathrm{pt} \stackrel{s}{\rightarrow} S$ defines an idempotent $\underline{\operatorname{Hom}}\left(S, e_{S}\right): \underline{\operatorname{Hom}}(S, \mathbb{Z} \mathrm{F}(Y \times S)) \rightarrow \underline{\operatorname{Hom}}(S, \mathbb{Z F}(Y \times S))$ in the category of $\mathbb{Z} \mathrm{F}_{*}$-presheaves. Set,

$$
\underline{\operatorname{Hom}}(S, \mathbb{Z} \mathrm{F}(Y \wedge(S, s))):=\operatorname{Ker}\left[\underline{\operatorname{Hom}}\left(S, e_{s}\right)\right] .
$$


Consider the idempotent $\underline{\operatorname{Hom}}\left(e_{s}, \underline{\operatorname{Hom}}(S, \mathbb{Z} \mathrm{F}(Y \wedge(S, s)))\right)$ of $\underline{\operatorname{Hom}}(S, \mathbb{Z} \mathrm{F}(Y \wedge(S, s)))$ in the category of $\mathbb{Z} \mathrm{F}_{*}(k)$-presheaves. Set,

$$
\underline{\operatorname{Hom}}((S, s), \mathbb{Z} \mathrm{F}(Y \wedge(S, s))):=\operatorname{Ker}\left[\underline{\operatorname{Hom}}\left(e_{s}, \underline{\operatorname{Hom}}(S, \mathbb{Z} \mathrm{F}(Y \wedge(S, s)))\right)\right] .
$$

For any $X \in S m / k$ denote by $\mathbb{Z F}(X \wedge(S, s), Y \wedge(S, s))$ the value of $\underline{\operatorname{Hom}}((S, s), \mathbb{Z F}(Y \wedge(S, s)))$ on $X$. There is a natural morphism of $\mathbb{Z} \mathrm{F}_{*}(k)$-presheaves

$$
-\otimes i d_{(S, s)}: \mathbb{Z F}(Y) \rightarrow \underline{\operatorname{Hom}}((S, s), \mathbb{Z} \mathrm{F}(Y \wedge(S, s))) .
$$

Definition 2.8. Let $X$ and $Y$ be $k$-smooth schemes and let $(S, s)$ be a $k$-smooth pointed scheme.

$\diamond$ Denote by $e_{s}: S \rightarrow \mathrm{pt} \stackrel{s}{\rightarrow} S$ the idempotent in $\operatorname{End}_{\mathbb{Z} \mathrm{F}_{0}(k)}(S)=\mathbb{Z F}_{0}(S, S)$ given by the composition of the constant map and the embedding of $s$ into $S$.

$\diamond$ For each integer $m \geqslant 0$ define $\mathbb{Z F}_{m}(X \wedge(S, s), Y \wedge(S, s))$ as a subgroup of the group $\mathbb{Z F}_{m}(X \times S, Y \times S)$ consisting of all $a$ such that $a \circ\left(\operatorname{id}_{X} \nabla e_{S}\right)=\left(\operatorname{id}_{Y} \bigotimes e_{S}\right) \circ a=0$. Note that the suspension map $\Sigma: \mathbb{Z}_{m}(X \times S, Y \times S) \rightarrow \mathbb{Z F}_{m+1}(X \times S, Y \times S)$ takes the subgroup $\left.\mathbb{Z F}_{m}(X \wedge(S, s), Y \wedge(S, s))\right)$ to the subgroup $\mathbb{Z F}_{m+1}(X \wedge(S, s), Y \wedge(S, s))$. Set,

$$
\mathbb{Z} \mathrm{F}(X \wedge(S, s), Y \wedge(S, s)):=\operatorname{colim}\left[\mathbb{Z} \mathrm{F}_{0}(X \wedge(S, s), Y \wedge(S, s)) \stackrel{\Sigma}{\rightarrow} \mathbb{Z}_{1}(X \wedge(S, s), Y \wedge(S, s)) \stackrel{\Sigma}{\rightarrow} \ldots\right],
$$

It is easy to see that the morphisms $i d_{X} \otimes\left(\operatorname{id}_{\mathbb{G}_{m}}-e_{s}\right): \mathbb{Z F}_{m}(X, Y) \rightarrow \mathbb{Z F}_{m}(X \times S, Y \times S)$ take values in $\mathbb{Z F}_{m}(X \wedge(S, s), Y \wedge(S, s))$. They are compatible with the suspension $\Sigma$ and we define a morphism

$$
i d_{X} \otimes \operatorname{id}_{(S, s)}: \mathbb{Z} \mathrm{F}(X, Y) \rightarrow \mathbb{Z} \mathrm{F}(X \wedge(S, s), Y \wedge(S, s)) .
$$

Lemma 2.9. Let $Y$ be $k$-smooth scheme and let $(S, s)$ be a $k$-smooth pointed scheme. Then one has a commutative diagram of $\mathbb{Z} \mathrm{F}_{*}(k)$-presheaves

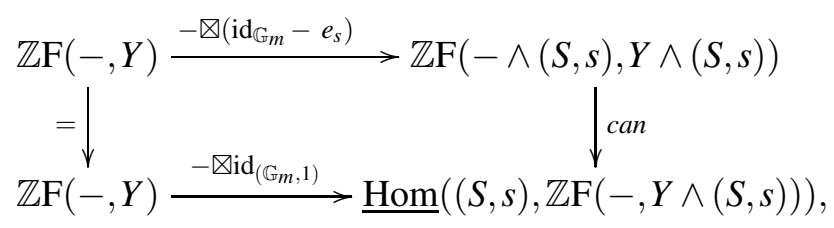

where can is the canonical isomorphism.

Theorem C. Let $X$ and $Y$ be $k$-smooth schemes and let $\left(\mathbb{G}_{m}, 1\right)$ be the scheme $\mathbb{G}_{m}$ pointed by the point 1 . Then the morphisms

$$
\begin{aligned}
& -\otimes\left(\operatorname{id}_{\mathbb{G}_{m}}-e_{1}\right): \mathbb{Z} \mathrm{F}\left(\Delta^{\bullet} \times-, Y\right) \rightarrow \mathbb{Z} \mathrm{F}\left(\left(\Delta^{\bullet} \times-\right) \wedge\left(\mathbb{G}_{m}, 1\right), Y \wedge\left(\mathbb{G}_{m}, 1\right)\right) \\
& -\otimes \operatorname{id}_{\left(\mathbb{G}_{m}, 1\right)}: \mathbb{Z} \mathrm{F}\left(\Delta^{\bullet} \times-, Y\right) \rightarrow \underline{\operatorname{Hom}}\left(\left(\mathbb{G}_{m}, 1\right), \mathbb{Z} \mathrm{F}\left(\Delta^{\bullet} \times-, Y \wedge\left(\mathbb{G}_{m}, 1\right)\right)\right)
\end{aligned}
$$

are sectionwise quasi-isomorphisms of complexes of $\mathbb{Z} \mathrm{F}_{*}(k)$-presheaves of abelian groups.

Remark 2.10. By Lemma 2.9 the morphism (1) is a quasi-isomorphism if and only if the morphism (2) is a quasi-isomorphism. Sometimes it is convenient to work with the morphism (1) and sometimes it is convenient to work with the morphism (2).

Theorem $\mathrm{C}$ will be proved at the end of the paper. 


\section{THEOREM A AND THEOREM B}

Before proving Theorem A we recall some definitions and constructions for framed motives for the convenience of the reader. We adhere to [GP1]. Let $\mathrm{Fr}_{0}(k)$ be the category whose objects are those of $S m / k$ and whose morphism set between $X$ and $Y$ is given by the set of framed correspondences of level zero [V1, Example 2.1], [GP1, Definition 2.1]. As it is shown in [GP1, Section 5], the category of framed correspondences of level zero $\operatorname{Fr}_{0}(k)$ has an action by finite pointed sets $Y \otimes K:=\bigsqcup_{K \backslash *} Y$ with $Y \in S m / k$ and $K$ a finite pointed set. Let $U, X \in \operatorname{Fr}_{0}(k)$. By the Additivity Theorem of [GP1] the $\Gamma$-space in the sense of Segal [Seg]

$$
K \in \Gamma^{\mathrm{op}} \mapsto C_{*} \operatorname{Fr}(U, X \otimes K):=\operatorname{Fr}\left(U \times \Delta^{\bullet}, X \otimes K\right)
$$

is special.

Definition 3.1 (see [GP1]). The framed motive $M_{f r}(X)$ of a smooth algebraic variety $X \in \mathrm{Fr}_{0}(k)$ is the Segal $S^{1}$-spectrum $\left(C_{*} \operatorname{Fr}(-, X), C_{*} \operatorname{Fr}\left(-, X \otimes S^{1}\right), C_{*} \operatorname{Fr}\left(-, X \otimes S^{2}\right), \ldots\right)$ associated with the special $\Gamma$-space $K \in \Gamma^{\mathrm{op}} \mapsto C_{*} \operatorname{Fr}(-, X \otimes K)$. The framed motive $M_{f r}(X) \in S p_{S^{1}}(k)$ is covariantly functorial in framed correspondences of level zero.

Let $\operatorname{Fr}_{0}(k)$ be the category whose objects are those of $S m / k$ and whose morphism set between $X$ and $Y$ is given by the set of framed correspondences of level zero [V1, Example 2.1], [GP1, Definition 2.1]. As it is shown in [GP1, Section 5], the category of framed correspondences of level zero $\operatorname{Fr}_{0}(k)$ has an action by finite pointed sets $Y \otimes K:=\bigsqcup_{K \backslash *} Y$ with $Y \in S m / k$ and $K$ a finite pointed set. The cone of $Y$ is the simplicial object $Y \otimes I$ in $\operatorname{Fr}_{0}(k)$, where $(I, 1)$ is the pointed simplicial set $\Delta[1]$ with basepoint 1 . There is a natural morphism $i_{0}: Y \rightarrow Y \otimes I$ in $\Delta^{\mathrm{op}} \operatorname{Fr}_{0}(k)$. Given a closed inclusion of smooth schemes $j: Y \hookrightarrow X$, denote by $X / / Y$ a simplicial object in $\operatorname{Fr}_{0}(k)$ which is obtained by taking the pushout of the diagram $X \hookleftarrow Y \stackrel{i_{0}}{\hookrightarrow} Y \otimes I$ in $\Delta^{\mathrm{op}} \operatorname{Fr}_{0}(k)$. The simplicial object $X / / Y$ termwise equals $X, X \sqcup Y, X \sqcup Y \sqcup Y, \ldots$. By $\mathbb{G}_{m}^{\wedge 1}$ we mean the simplicial object $\mathbb{G}_{m} / /\{1\}$ in $\operatorname{Fr}_{0}(k)$. It looks termwise as

$$
\mathbb{G}_{m}, \mathbb{G}_{m} \sqcup p t, \mathbb{G}_{m} \sqcup p t \sqcup p t, \ldots
$$

Applying $M_{f r}(X \times-)$ to $\mathbb{G}_{m}^{\wedge 1}$ and realizing by taking diagonals, one gets a framed $S^{1}$-spectrum $M_{f r}\left(X \times \mathbb{G}_{m}^{\wedge 1}\right)$. We shall also denote it by $M_{f r}(X)(1)$. The $n$th iteration gives the spectrum $M_{f r}(X \times$ $\left.\mathbb{G}_{m}^{\wedge n}\right)$, also denoted by $M_{f r}(X)(n)$.

Similarly to the General Framework on p. 6 there is a natural pairing

$$
\nabla: s \operatorname{Pre}_{\bullet}^{f r}(k) \times s \operatorname{Pre}_{\bullet}\left(\mathrm{Fr}_{0}(k)\right) \rightarrow s \operatorname{Pre}_{\bullet}^{f r}(k),
$$

where $s \operatorname{Pre}_{\bullet}^{f r}(k)$ (respectively $\left.s \operatorname{Pre}_{\bullet}\left(\operatorname{Fr}_{0}(k)\right)\right)$ is the category of pointed simplicial presheaves with framed correspondences (respectivley the category of pointed simplicial presheaves on $\operatorname{Fr}_{0}(k)$ ). It is extended from the pairing $\operatorname{Fr}_{*}(k) \times \mathrm{Fr}_{0}(k) \stackrel{\otimes}{\rightarrow} \mathrm{Fr}_{*}(k)$ that takes $(X, Y)$ to $X \times Y$ and $a \in \mathrm{Fr}_{m}\left(X, X^{\prime}\right)$, $b \in \operatorname{Fr}_{0}\left(Y, Y^{\prime}\right)$ to $a \bigotimes b \in \operatorname{Fr}_{m}\left(X \times X^{\prime}, Y \times Y^{\prime}\right)$.

We will also write $\wedge$ for the monoidal product in $\operatorname{Fr}_{0}(k)$ and in $\Delta^{\mathrm{op}} \mathrm{Fr}_{0}(k)$. The Yoneda embedding identifies $\Delta^{\mathrm{op}} \mathrm{Fr}_{0}(k)$ with a full subcategory of $s \operatorname{Pre}_{\bullet}\left(\operatorname{Fr}_{0}(k)\right)$. For each integer $n \geqslant 0$ there is a natural map of spectra

$$
a_{n}: M_{f r}\left(X \times \mathbb{G}_{m}^{\wedge n}\right) \stackrel{-\unrhd \mathbb{G}_{m}^{\wedge 1}}{\longrightarrow} \underline{\operatorname{Hom}}\left(\mathbb{G}_{m}^{\wedge 1}, M_{f r}\left(X \times \mathbb{G}_{m}^{\wedge n+1}\right)\right) \rightarrow \underline{\operatorname{Hom}}\left(\mathbb{G}, M_{f r}\left(X \times \mathbb{G}_{m}^{\wedge n+1}\right)\right),
$$

where the right arrow is induced by the adjunction unit adj : $\mathbb{G} \rightarrow\left(\left.\mathbb{G}_{m}^{\wedge 1}\right|_{S m / k}\right)$. Note that $a_{n}$ respects framed correspondences of level zero and coincides with the morphism described in [GP1, p. 297]). 
Definition 3.2. The $\left(S^{1}, \mathbb{G}\right)$-bispectrum $M_{f r}^{\mathbb{G}}(X)$ is defined as

$$
\left(M_{f r}(X), M_{f r}\left(X \times \mathbb{G}_{m}^{\wedge 1}\right), M_{f r}\left(X \times \mathbb{G}_{m}^{\wedge 2}\right), \ldots\right)
$$

together with the structure morphisms $a_{n}$-s.

We shall prove below (see the proof of Theorem A) that each $a_{n}$ is a schemewise stable equivalence of spectra, but first let us discuss further useful spectra. Denote by $\mathbb{Z} M_{f r}(X), X \in S m / k$, the Segal $S^{1}$-spectrum $\left(\mathbb{Z F r}\left(\Delta^{\bullet} \times-, X\right), \mathbb{Z F r}\left(\Delta^{\bullet} \times-, X \otimes S^{1}\right), \ldots\right)$. Denote by $L M_{f r}(X)$ the Segal $S^{1}$-spectrum $E M\left(\mathbb{Z F}\left(\Delta^{\bullet} \times-, X\right)\right)=\left(\mathbb{Z F}\left(\Delta^{\bullet} \times-, X\right), \mathbb{Z} \mathrm{F}\left(\Delta^{\bullet} \times-, X \otimes S^{1}\right), \ldots\right)$.

The equalities $\mathbb{Z} \mathrm{F}\left(-, X \sqcup X^{\prime}\right)=\mathbb{Z} \mathrm{F}(-, X) \oplus \mathbb{Z} \mathrm{F}\left(-, X^{\prime}\right)$ show that the $\Gamma$-space $(K, *) \mapsto \mathbb{Z} \mathrm{F}\left(\Delta^{\bullet} \times\right.$ $U, X \otimes K)$ corresponds to the complex of abelian groups $\mathbb{Z} \mathrm{F}\left(\Delta^{\bullet} \times U, X\right)$. Hence $L M_{f r}(X)$ is the Eilenberg-Mac Lane spectrum for the complex $\mathbb{Z F}\left(\Delta^{\bullet} \times-, X\right)$. The $\Gamma$-space morphism

$$
\left[(K, *) \mapsto \mathbb{Z} \mathrm{Fr}\left(\Delta^{\bullet} \times-, X \otimes K\right)\right] \rightarrow\left[(K, *) \mapsto \mathbb{Z} \mathrm{F}\left(\Delta^{\bullet} \times-, X \otimes K\right)\right]
$$

induces a morphism of $S^{1}$-spectra $l_{X}: \mathbb{Z} M_{f r}(X) \rightarrow E M(\mathbb{Z F}(-, X))$.

Note that homotopy groups of $L M_{f r}(X)=E M\left(\mathbb{Z} \mathrm{F}\left(\Delta^{\bullet} \times-, X\right)\right)$ are equal to homology groups of the complex $\mathbb{Z} \mathrm{F}\left(\Delta^{\bullet} \times-, X\right)$. By [Sch, $\S$ II.6.2] the homotopy groups $\pi_{*}\left(\mathbb{Z} M_{f r}(X)(U)\right)$ of $\mathbb{Z} M_{f_{r}}(X)$ evaluated at $U \in S m / k$ are the homology groups $H_{*}\left(M_{f r}(X)(U)\right)$ of $M_{f r}(X)(U)$.

The following result, referred to as the Linearisation Theorem in [GNP, Theorem 1.2], is true:

Theorem 3.3 (see [GNP]). The morphism of $S^{1}$-spectra

$$
l_{X}: \mathbb{Z} M_{f r}(X) \rightarrow L M_{f r}(X)
$$

is a schemewise stable equivalence. In particular, if $U$ is smooth, then

$$
H_{*}\left(M_{f r}(X)(U)\right)=\pi_{*}\left(\mathbb{Z} M_{f r}(X)(U)\right)=\pi_{*}\left(L M_{f r}(X)(U)\right)=H_{*}\left(\mathbb{Z F}\left(\Delta^{\bullet} \times U, X\right)\right) .
$$

Replacing simplicial framed sheaves $C_{*} F r$ in Definition 3.2 by simplicial abelian framed presheaves $C_{*} \mathbb{Z F}$, we define Segal $S^{1}$-spectra $L M_{f r}\left(X \times \mathbb{G}_{m}^{\wedge n}\right)$-s. Following the General Framework on p. 6, there is a natural morphism of $S^{1}$-spectra for each integer $n \geqslant 0$

$$
c_{n}: L M_{f r}\left(X \times \mathbb{G}_{m}^{\wedge n}\right) \stackrel{-\otimes \mathbb{G}_{m}^{\wedge 1}}{\longrightarrow} \underline{\operatorname{Hom}}\left(\mathbb{G}_{m}^{\wedge 1}, M_{f r}\left(X \times \mathbb{G}_{m}^{\wedge n+1}\right)\right) \rightarrow \underline{\operatorname{Hom}}\left(\mathbb{G}, L M_{f r}\left(X \times \mathbb{G}_{m}^{\wedge n+1}\right)\right),
$$

where the right arrow is induced by the adjunction unit adj : $\mathbb{G} \rightarrow\left(\left.\mathbb{G}_{m}^{\wedge 1}\right|_{S m / k}\right)$.

Definition 3.4. The $\left(S^{1}, \mathbb{G}\right)$-bispectrum $L M_{f r}^{\mathbb{G}}(X)$ is defined as

$$
\left(L M_{f r}(X), L M_{f r}\left(X \times \mathbb{G}_{m}^{\wedge 1}\right), L M_{f r}\left(X \times \mathbb{G}_{m}^{\wedge 2}\right), \ldots\right)
$$

together with the structure morphisms $c_{n}$-s.

We are now in a position to prove Theorem A.

Proof of Theorem A. (1). We claim that for every $n>0$ the sequence

$$
M_{f r}(X)(n-1) \rightarrow M_{f r}\left(X \times \mathbb{G}_{m}\right)(n-1) \rightarrow M_{f r}(X)(n)
$$

is a homotopy cofiber sequence of $S^{1}$-spectra. Since all spectra are connected, it is enough to show that

$$
\mathbb{Z} M_{f r}(X)(n-1) \rightarrow \mathbb{Z} M_{f r}\left(X \times \mathbb{G}_{m}\right)(n-1) \rightarrow \mathbb{Z} M_{f r}(X)(n)
$$

is a homotopy cofiber sequence of $S^{1}$-spectra. By Theorem 3.3 the latter is equivalent to showing that

$$
L M_{f r}(X)(n-1) \rightarrow L M_{f r}\left(X \times \mathbb{G}_{m}\right)(n-1) \rightarrow L M_{f r}(X)(n)
$$


is a homotopy cofiber sequence of $S^{1}$-spectra. This sequence is a homotopy cofiber sequence if and only if

$$
\mathbb{Z} F\left(\Delta^{\bullet} \times-, X \times \mathbb{G}_{m}^{\wedge(n-1)}\right) \rightarrow \mathbb{Z} F\left(\Delta^{\bullet} \times-, X \times \mathbb{G}_{m}^{\wedge(n-1)} \times \mathbb{G}_{m}\right) \rightarrow \mathbb{Z} F\left(\Delta^{\bullet} \times-, X \times \mathbb{G}_{m}^{\wedge n}\right)
$$

is a homotopy cofiber sequence of complexes of abelian presheaves. But this is obvious because $\mathbb{Z} F\left(\Delta^{\bullet} \times-, X \times \mathbb{G}_{m}^{\wedge n}\right)$ is the mapping cone of the left arrow, and hence the desired claim follows. We have used here the fact that $\mathbb{Z} F(-, X \sqcup Y)=\mathbb{Z} F(-, X) \oplus \mathbb{Z} F(-, Y)$.

Next, it is enough to prove that

$$
a_{0}: M_{f r}(X) \rightarrow \underline{\operatorname{Hom}}\left(\mathbb{G}, M_{f r}(X)(1)\right)
$$

is a schemewise equivalence of spectra. Indeed, consider a commutative diagram of homotopy cofiber sequences in $S p_{S^{1}}(k)$

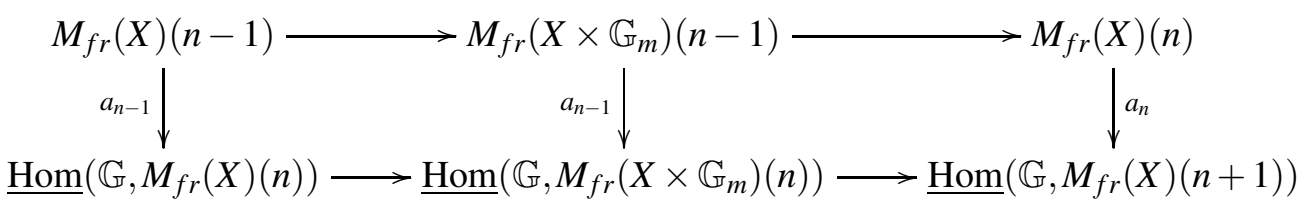

with $n \geqslant 1$. If $a_{n-1}$ is a schemewise equivalence of spectra, then so is $a_{n}$ by [Hir, 13.5.10]. Thus using induction in $n$, it suffices to verify that $a_{0}$ is a schemewise equivalence of spectra.

By the stable Whitehead theorem [Sch, II.6.30] $a_{0}$ is a stable equivalence if and only if so is

$$
a_{0}: \mathbb{Z} M_{f r}(X) \rightarrow \mathbb{Z}\left[\underline{\operatorname{Hom}}\left(\mathbb{G}, M_{f r}\left(X \times \mathbb{G}_{m}^{\wedge 1}\right)\right)\right] .
$$

Consider a commutative diagram of homotopy fiber sequences in $S p_{S^{1}}(k)$

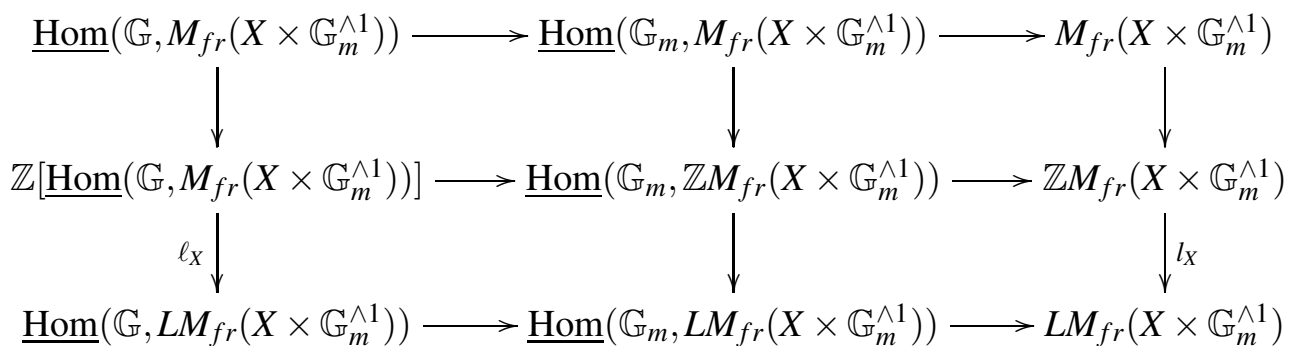

The arrow $l_{X}$ and the middle lower arrow are a stable weak equivalences of spectra by Theorem 3.3. It follows that $\ell_{X}$ is a stable weak equivalence. Consider a commutative diagram

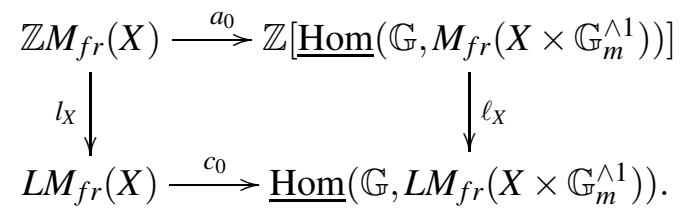

Since $l_{X}, \ell_{X}$ are stable weak equivalences, it follows that $a_{0}$ is a stable local equivalence if and only if so is $c_{0}$. By Theorem D from Appendix A the morphism $c_{0}$ is a sectionwise stable weak equivalence. The proof of the first part of the theorem is completed.

(2). Since each spectrum $M_{f r}(X)(n)_{f}$ is fibrant in the injective local stable model structure of $S^{1}$-spectra, it is enough to show that each map

$$
b_{n}: M_{f r}(X)(n)_{f} \rightarrow \underline{\operatorname{Hom}}\left(\mathbb{G}, M_{f r}(X)(n+1)_{f}\right), \quad n \geqslant 0,
$$


is a Nisnevich local stable equivalence of spectra. Using the same argument as in the proof of the first statement, it suffices to verify that $b_{0}$ is a local stable equivalence.

There is a commutative diagram

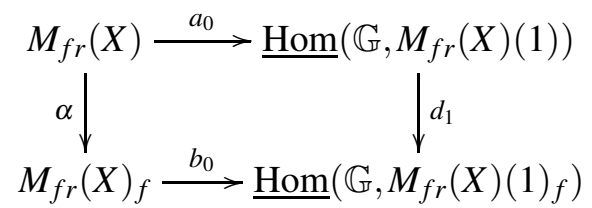

in which the left vertical arrow is a local stable equivalence and $a_{0}$ is a schemewise stable equivalence by the first statement. It follows that $b_{0}$ is a local stable equivalence if and only if so is $d_{1}=\underline{\operatorname{Hom}}(\mathbb{G}, \alpha)$.

The presheaves of stable homotopy groups of $\underline{\operatorname{Hom}}\left(\mathbb{G}, M_{f r}(X)(1)\right)$ equal $\left(\pi_{n}\left(M_{f r}(X)(1)\right)\right)_{-1}$. These presheaves are $\mathbb{A}^{1}$-invariant quasi-stable radditive with framed correspondences (see [GP2, Introduction] for the definition of such presheaves). It follows from [GP2, Theorem 1.1] (complemented by [DP] in characteristic 2) that each Nisnevich sheaf $\left(\left(\pi_{n}\left(M_{f r}(X)(1)\right)\right)_{-1}\right)^{\text {nis }}$ is strictly $\mathbb{A}^{1}$-invariant quasi-stable radditive with framed correspondences.

Each spectrum $M_{f r}(X)(n)$ has homotopy invariant, quasi-stable radditive presheaves with framed correspondences of stable homotopy groups $\pi_{*}\left(M_{f r}(X)(n)\right)$. By [GP2, Theorem 1.1] (complemented by [DP] in characteristic 2) the Nisnevich sheaves $\pi_{*}^{\text {nis }}\left(M_{f r}(X)(n)\right)$ are strictly homotopy invariant. It follows from [GP1, Proposition 7.1] that $M_{f r}(X)(n)_{f}$ is motivically fibrant in the injective stable motivic model structure of $S^{1}$-spectra.

In order to compute the Nisnevich sheaf $\pi_{n}^{\text {nis }}\left(\underline{\operatorname{Hom}}\left(\mathbb{G}, M_{f r}(X)(1)_{f}\right)\right)$, consider the BrownGersten convergent spectral sequence

$$
H_{\mathrm{nis}}^{p}\left(V \times \mathbb{G}_{m}, \pi_{q}^{\mathrm{nis}}\left(M_{f r}(X)(1)\right)\right) \Rightarrow \pi_{q-p}\left(M_{f r}(X)(1)_{f}\left(V \times \mathbb{G}_{m}\right)\right), \quad V \in S m / k .
$$

It follows from [GP2, Corollary 16.8, Theorems 17.15-16] that each presheaf

$$
V \mapsto H_{\text {nis }}^{p}\left(V \times \mathbb{G}_{m}, \pi_{q}^{\mathrm{nis}}\left(M_{f r}(X)(1)\right)\right)
$$

is $\mathbb{A}^{1}$-invariant quasi-stable radditive with framed correspondences.

Let $V \in S m / k$ be irreducible, $u \in V$ be a point, $U=\operatorname{Spec}\left(\mathscr{O}_{V, u}\right)$. Let $U_{u}^{h}$ be the henselization of $U$ at $u$ and let $k\left(U_{u}^{h}\right)$ be the function field on $U_{u}^{h}$. Consider the above spectral sequence and replace $V$ by $U_{u}^{h}$ in it. We claim that in this case the spectral sequence degenerates and $H_{\text {nis }}^{0}\left(U_{u}^{h} \times \mathbb{G}_{m}, \pi_{n}^{\text {nis }}\left(M_{f r}(X)(1)\right)\right)=\pi_{n}\left(M_{f r}(X)(1)_{f}\left(U_{u}^{h} \times \mathbb{G}_{m}\right)\right)$. For this notice that by [GP2, 3.15(3')] the map $H_{\text {nis }}^{p}\left(\mathbb{G}_{m} \times U, \pi_{q}^{\text {nis }}\left(M_{f r}(X)(1)\right)\right) \hookrightarrow H_{\text {nis }}^{p}\left(\mathbb{G}_{m, k\left(U_{u}^{h}\right)}, \pi_{q}^{\text {nis }}\left(M_{f r}(X)(1)\right)\right)$ is injective, where $\eta_{h}: \operatorname{Spec}\left(k\left(U_{u}^{h}\right)\right) \rightarrow U_{u}^{h}$ is the canonical morphism. In turn, by [GP2, 3.15(1)] the canonical homomorphism

$$
H_{\text {nis }}^{p}\left(\mathbb{G}_{m, k\left(U_{u}^{h}\right)}, \pi_{q}^{\mathrm{nis}}\left(M_{f r}(X)(1)\right)\right) \hookrightarrow H_{\mathrm{nis}}^{p}\left(\operatorname{Spec}\left(k\left(U_{u}^{h}\right)(t)\right), \pi_{q}^{\mathrm{nis}}\left(M_{f r}(X)(1)\right)\right)
$$

is injective. Since $0=H_{\text {nis }}^{p}\left(\operatorname{Spec}\left(k\left(U_{u}^{h}\right)(t)\right), \pi_{q}^{\text {nis }}\left(M_{f r}(X)(1)\right)\right)$ for $p>0$, the group $H_{\text {nis }}^{p}\left(U_{u}^{h} \times\right.$ $\left.\mathbb{G}_{m}, \pi_{q}^{\text {nis }}\left(M_{f r}(X)(1)\right)\right)$ vanishes for $p>0$. Thus we have checked the equality

$$
H_{\mathrm{nis}}^{0}\left(U_{u}^{h} \times \mathbb{G}_{m}, \pi_{n}^{\mathrm{nis}}\left(M_{f r}(X)(1)\right)\right)=\pi_{n}\left(M_{f r}(X)(1)_{f}\left(U_{u}^{h} \times \mathbb{G}_{m}\right)\right) .
$$

We can conclude that $\pi_{n}^{\text {nis }}\left(\underline{\operatorname{Hom}}\left(\mathbb{G}_{m}, M_{f r}(X)(1)_{f}\right)\right)=\pi_{n}^{\text {nis }}\left(M_{f r}(X)(1)_{f}\right)\left(\mathbb{G}_{m} \times-\right)$. It follows that

$$
\pi_{n}^{\mathrm{nis}}\left(\underline{\operatorname{Hom}}\left(\mathbb{G}, M_{f r}(X)(1)_{f}\right)\right)=\left(\pi_{n}^{\mathrm{nis}}\left(M_{f r}(X)(1)_{f}\right)\right)_{-1}=\left(\pi_{n}^{\mathrm{nis}}\left(M_{f r}(X)(1)\right)\right)_{-1} .
$$


It remains to show that the morphism of $\mathbb{A}^{1}$-invariant radditive quasi-stable framed sheaves

$$
\left(\left(\pi_{n}\left(M_{f r}(X)(1)\right)\right)_{-1}\right)^{\mathrm{nis}} \rightarrow\left(\pi_{n}^{\mathrm{nis}}\left(M_{f r}(X)(1)\right)\right)_{-1}
$$

is an isomorphism. Using [GP2, 3.15(3')] it suffices to check that it is an isomorphism for every field extension $K / k$. The homomorphism of abelian groups

$$
\left(\left(\pi_{n}\left(M_{f r}(X)(1)\right)\right)_{-1}\right)^{\mathrm{nis}}(K)=\left(\pi_{n}\left(M_{f r}(X)(1)\right)\right)_{-1}(K) \rightarrow\left(\pi_{n}^{\mathrm{nis}}\left(M_{f r}(X)(1)\right)\right)_{-1}(K)
$$

is an isomorphism, because for every homotopy invariant radditive quasi-stable framed presheaf of abelian groups $\mathscr{F}$ and every open $V \subset \mathbb{A}_{K}^{1}$, one has $\mathscr{F}(V)=\mathscr{F}^{\text {nis }}(V)$ (see the proof of $[\mathrm{GP} 2,3.1]$ ). This completes the proof of Theorem A.

Proof of Theorem B. The proof of Theorem A(2) shows that $M_{f r}(X)(n)_{f}$ is motivically fibrant in the injective stable motivic model structure of $S^{1}$-spectra. By Theorem A each structure map $b_{n}$ is a schemewise equivalence. We conclude that the bispectrum $M_{f r}^{\mathbb{G}}(X)_{f}$ is a motivically fibrant $\left(S^{1}, \mathbb{G}\right)$-bispectrum in the sense of Jardine [Jar].

\section{USEFUL LEMMAS}

In this section we discuss several useful $\mathbb{A}^{1}$-homotopies and collect a number of facts used in the following sections. We start with some definitions and notation.

Definition 4.1. Let $\mathscr{F}: S m / k \rightarrow$ Sets be a presheaf of sets. Let $X \in S m / k$ be a smooth variety and $a, b \in \mathscr{F}(X)$ be two sections. We write $a \sim b$ if $a$ and $b$ are in the same connected component of the simplicial set $\mathscr{F}\left(\Delta^{\bullet} \times X\right)$. If $h \in \mathscr{F}\left(\Delta^{1} \times X\right)$ is such that $\partial_{0}(h)=a$ and $\partial_{1}(h)=b$, then we will write $a^{\underline{h}} b$. In this case $a \sim b$.

Let $\mathscr{A}: S m / k \rightarrow A b$ be a presheaf of abelian groups. Let $X \in S m / k$ be a smooth variety and $a, b \in \mathscr{A}(X)$ be two sections. We will write $a \sim b$ if the classes of $a$ and $b$ in $H_{0}\left(\mathscr{A}\left(\Delta^{\bullet} \times X\right)\right)$ coincide. This is equivalent to saying that there is $h \in \mathscr{A}\left(\Delta^{1} \times X\right)$ such that $\partial_{0}(h)=a$ and $\partial_{1}(h)=b$. For such an $h$ we will write $a^{\underline{h}} b$.

Definition 4.2. Let $\mathscr{F}$ and $\mathscr{G}$ be two presheaves of sets on the category of $k$-smooth schemes and let $\varphi_{0}, \varphi_{1}: \mathscr{F} \rightrightarrows \mathscr{G}$ be two morphisms. An $\mathbb{A}^{1}$-homotopy between $\varphi_{0}$ and $\varphi_{1}$ is a morphism $H: \mathscr{F} \rightarrow \underline{\operatorname{Hom}}\left(\mathbb{A}^{1}, \mathscr{G}\right)$ such that $H_{0}=\varphi_{0}$ and $H_{1}=\varphi_{1}$. We will write $\varphi_{0} \sim \varphi_{1}$ if there is an $\mathbb{A}^{1}$ homotopy between $\varphi_{0}$ and $\varphi_{1}$.

Let $\mathscr{A}$ and $\mathscr{B}$ be two presheaves of abelian groups on the category of $k$-smooth schemes and let $\varphi_{0}, \varphi_{1}: \mathscr{A} \rightrightarrows \mathscr{B}$ be two morphisms. An $\mathbb{A}^{1}$-homotopy between $\varphi_{0}$ and $\varphi_{1}$ is a morphism $H$ : $\mathscr{A} \rightarrow \underline{\operatorname{Hom}}\left(\mathbb{A}^{1}, \mathscr{B}\right)$ of presheaves of abelian groups such that $H_{0}=\varphi_{0}$ and $H_{1}=\varphi_{1}$. If $H$ is an $\mathbb{A}^{1}$-homotopy between $\varphi_{0}$ and $\varphi_{1}$, then we will write $\varphi_{0} \underline{H} \varphi_{1}$. If we do not specify an $\mathbb{A}^{1}$-homotopy between $\varphi_{0}$ and $\varphi_{1}$, then we will write $\varphi_{0} \sim \varphi_{1}$.

If $\varphi: \mathscr{A} \rightarrow \mathscr{B}$ is a morphism of presheaves of abelian groups, then there is a constant $\mathbb{A}^{1}$ homotopy $H_{\varphi}$ between $\varphi$ and $\varphi$ defined as follows. Given $a \in \mathscr{A}(X)$ set $H_{\varphi}(a)=\operatorname{pr}_{X}^{*}(\varphi(a)) \in$ $\mathscr{B}\left(X \times \mathbb{A}^{1}\right)$.

Lemma 4.3. Let $\mathscr{A}$ and $\mathscr{B}$ be two presheaves of abelian groups on the category of $k$-smooth schemes and let $\varphi_{0}, \varphi_{1}: \mathscr{A} \rightrightarrows \mathscr{B}$ be two morphisms such that $\varphi_{0} \sim \varphi_{1}$. Then the induced morphisms

$$
\varphi_{0}, \varphi_{1}: \mathscr{A}\left(\Delta^{\bullet}\right) \rightrightarrows \mathscr{B}\left(\Delta^{\bullet}\right)
$$

between two simplicial abelian groups give the same morphisms on the homology of the associated Moore complexes. 
Lemma 4.4. Let $\varphi_{0}, \varphi_{1}, \varphi_{2}: \mathscr{A} \rightarrow \mathscr{B}$ be morphisms of presheaves of abelian groups and let $\varphi_{0} \frac{H^{\prime}}{\varphi_{1}}$ and $\varphi_{1} \stackrel{H^{\prime \prime}}{\varphi_{2}}$. Then

$$
\varphi_{0} \stackrel{H^{\prime}+H^{\prime \prime}-H_{\varphi_{1}}}{\varphi_{2}}
$$

Lemma 4.5. Let $\mathscr{A}$ and $\mathscr{B}$ be two presheaves of abelian groups on the category of $k$-smooth schemes and let $\varphi_{0} \underline{H} \varphi_{1}$. Let $\rho: \mathscr{A}^{\prime} \rightarrow \mathscr{A}$ be a morphism. Then $\varphi_{0} \circ \rho \frac{H \circ \rho}{} \varphi_{1} \circ \rho$. Moreover, let $\eta: \mathscr{B} \rightarrow \mathscr{B}^{\prime}$ be a morphism, then $\psi \circ \varphi_{0} \frac{\psi \circ H}{\psi} \psi \varphi_{1}$ with $\psi=\underline{\operatorname{Hom}}\left(\mathbb{A}^{1}, \eta\right): \underline{\operatorname{Hom}}\left(\mathbb{A}^{1}, \mathscr{B}\right) \rightarrow$ $\underline{\operatorname{Hom}}\left(\mathbb{A}^{1}, \mathscr{B}^{\prime}\right)$.

We now want to discuss actions of matrices on framed correspondences and associated homotopies. Let $X$ and $Y$ be $k$-smooth schemes and $A \in G L_{n}(k)$ be a matrix. Then $A$ defines an automorphism

$$
\varphi_{A}: \mathrm{Fr}_{n}(-\times X, Y) \rightarrow \mathrm{Fr}_{n}(-\times X, Y)
$$

of the presheaf $\operatorname{Fr}_{n}(-\times X, Y)$ in the following way. Given $W \in S m / k$ and $a=$ $\left(Z, U,\left(\varphi_{1}, \varphi_{2}, \ldots, \varphi_{n}\right), g\right) \in \operatorname{Fr}_{n}(W \times X, Y)$, set

$$
\left.\varphi_{A}\left(Z, U,\left(\varphi_{1}, \varphi_{2}, \ldots, \varphi_{n}\right), g\right)\right):=\left(Z, U, A \circ\left(\varphi_{1}, \varphi_{2}, \ldots, \varphi_{n}\right), g\right),
$$

where $A$ is regarded as a linear automorphism of $\mathbb{A}_{k}^{n}$.

The automorphism $\varphi_{A}$ of the presheaf $\operatorname{Fr}_{n}(-\times X, Y)$ induces an automorphism of the free abelian presheaf $\mathbb{Z}\left[\operatorname{Fr}_{n}(-\times X, Y)\right]$ and an automorphism $\varphi_{A}$ of the presheaf of abelian groups $\mathbb{Z} \mathrm{F}_{n}(-\times$ $X, Y)$.

Definition 4.6. Let $A \in S L_{n}(k)$. Choose a matrix $A_{s} \in S L_{n}(k[s])$ such that $A_{0}=i d$ and $A_{1}=A$. The matrix $A_{s}$, regarded as a morphism $\mathbb{A}^{n} \times \mathbb{A}^{1} \rightarrow \mathbb{A}^{n}$, gives rise to an $\mathbb{A}^{1}$-homotopy $h$ between $i d$ and $\varphi_{A}$ as follows. Given $\left.a=(\alpha, f, Z, U, \varphi, g)\right)=\left(\left(\alpha_{1}, \alpha_{2}, \ldots, \alpha_{n}\right), f, Z, U,\left(\varphi_{1}, \varphi_{2}, \ldots, \varphi_{n}\right), g\right) \in$ $\mathrm{Fr}_{n}(W \times X, Y)$, one sets

$$
h(a)=\left(\alpha, f \times i d_{\mathbb{A}^{1}}, Z \times \mathbb{A}^{1}, U \times \mathbb{A}^{1}, A_{s} \circ\left(\varphi \times i d_{\mathbb{A}^{1}}\right), g \circ p r_{U}\right) \in \operatorname{Fr}_{n}\left(W \times X \times \mathbb{A}^{1}, Y\right) .
$$

Clearly, $h_{0}(a)=a$ and $h_{1}(a)=\varphi_{A}(a)$. By linearity the homotopy $h$ induces an $\mathbb{A}^{1}$-homotopy $H_{A_{s}}$

$$
i d \stackrel{H_{A_{s}}}{ } \varphi_{A}: \mathbb{Z F}_{n}(-\times X, Y) \rightrightarrows \mathbb{Z F}_{n}(-\times X, Y)
$$

between the identity $i d$ and the morphism $\varphi_{A}$.

Lemma 4.7. Let $\rho: \mathbb{Z}_{m}(-\times X, Y) \rightarrow \mathbb{Z}_{n}(-\times X, Y)$ be a presheaf morphism. Let $A \in S L_{n}(k)$, $A_{s} \in S L_{n}(k[s])$ and $H_{A_{s}}$ be as in Definition 4.6. Then one has

$$
\rho \frac{H_{A_{s}} \circ \rho}{} \varphi_{A} \circ \rho: \mathbb{Z F}_{m}(-\times X, Y) \rightrightarrows \mathbb{Z} \mathrm{F}_{n}(-\times X, Y) .
$$

For $b \in \mathbb{Z F}_{m}(Y, S)$ define a presheaf morphism

$$
\varphi_{b}: \mathbb{Z F}_{n}(-\times X, Y) \rightarrow \mathbb{Z F}_{n+m}(-\times X, S)
$$

sending $a \in \mathbb{Z F}_{n}(W \times X, Y)$ to $b \circ a \in \mathbb{Z F}_{n+m}(W \times X, S)$. Also, any $b \in \mathbb{Z F}_{m}(p t, p t)$ defines a morphism of presheaves

$$
-\otimes b: \mathbb{Z} \mathrm{F}_{n}(-\times X, Y) \rightarrow \mathbb{Z}_{n+m}(-\times X, Y)
$$

sending $a \in \mathbb{Z F}_{n}(W \times X, Y)$ to $a \bigotimes b \in \mathbb{Z} \mathrm{F}_{n+m}(W \times X, Y)$.

The next three lemmas are straightforward. 
Lemma 4.8. Let $b_{1}, b_{2} \in \mathbb{Z F}_{m}(Y, S)$ be such that $b_{1} \sim b_{2}$, then

$$
\varphi_{b_{1}} \sim \varphi_{b_{2}}: \mathbb{Z F}_{n}(-\times X, Y) \rightrightarrows \mathbb{Z F}_{n+m}(-\times X, S) .
$$

Lemma 4.9. Let $b_{1}, b_{2} \in \mathbb{Z} \mathrm{F}_{m}(p t, p t)$ and $h \in \mathbb{Z F}_{m}\left(\mathbb{A}^{1}\right.$,pt $)$ be such that $b_{1} \underline{h} b_{2}$, then

$$
\left(-\otimes b_{1}\right) \stackrel{-\otimes h}{-}\left(-\otimes b_{2}\right): \mathbb{Z F}_{n}(-\times X, Y) \rightrightarrows \mathbb{Z F}_{n+m}(-\times X, Y) .
$$

The following lemma is proved in Appendix B.

Lemma 4.10. Let $z \in \mathbb{A}^{m}$ be a $k$-rational point. Set $U^{\prime}=\left(\mathbb{A}^{m}\right)_{z}^{h}$ to be the henzelization of $\mathbb{A}^{m}$ at the point $z$. Let $i_{z}: \mathrm{pt} \hookrightarrow U^{\prime}$ be the closed point of $U^{\prime}$. Let $U_{s}^{\prime}:=\left(\mathbb{A}^{1} \times \mathbb{A}^{m}\right)_{\mathbb{A}^{1} \times z}^{h}$ be the henzelization of $\mathbb{A}^{1} \times \mathbb{A}^{m}$ at $\mathbb{A}^{1} \times z$. Then the morphism $f_{s}: \mathbb{A}^{1} \times \mathbb{A}^{m} \rightarrow \mathbb{A}^{m}$ mapping $(s, y)$ to $s \cdot(y-x)+x$ induces a morphism $H_{s}:=f_{s}^{h}: U_{s}^{\prime} \rightarrow U^{\prime}$ such that:

(a) $H_{1}:=\left.\left(f_{s}^{h}\right)\right|_{(1 \times X)_{(1, x)}^{h}}: U^{\prime} \rightarrow U^{\prime}$ is the identity morphism;

(b) $H_{0}:=\left.\left(f_{s}^{h}\right)\right|_{(0 \times X)_{(0, x)}^{h}}: U^{\prime} \rightarrow U^{\prime}$ coincides with the composite morphism $U^{\prime} \stackrel{p^{h}}{\rightarrow} \mathrm{pt} \stackrel{s_{z}}{\rightarrow} U^{\prime}$, where $p^{h}: U^{\prime} \rightarrow \mathrm{pt}=\operatorname{Spec}(k)$ is the structure morphism and $s_{z}: \mathrm{pt} \hookrightarrow U^{\prime}$ is the closed point of $U^{\prime}$.

Let $z \in \mathbb{A}^{m}$ be a $k$-rational point. The projection $p r: \mathbb{A}^{1} \times \mathbb{A}^{m} \rightarrow \mathbb{A}^{m}$ induces a morphism $\operatorname{can}_{s}:=$ $p r^{h}: U_{s}^{\prime} \rightarrow U^{\prime}$ such that $c a n_{0}=c a n_{1}=\mathrm{id}_{U^{\prime}}($ see Appendix B). The preceding lemma gives the following

Corollary 4.11. Let $z \in \mathbb{A}^{m}$ be a $k$-rational point and let $\left(z, U^{\prime}, \psi ; \mathrm{id}_{U^{\prime}}\right) \in \mathrm{Fr}_{m}\left(\mathrm{pt}, U^{\prime}\right)$ with $U^{\prime}$ as in Lemma 4.10. Suppose $U_{s}^{\prime}$ is as in Lemma 4.10 and let $h_{s}=\left(\mathbb{A}^{1} \times z, U_{s}^{\prime}, \operatorname{can}_{s}^{*}(\psi) ; H_{s}\right) \in \operatorname{Fr}_{m}\left(\mathbb{A}^{1}, U^{\prime}\right)$. Then one has:

(a) $h_{1}=\left(z, U^{\prime}, \psi ; \operatorname{id}_{U^{\prime}}\right) \in \mathrm{Fr}_{m}\left(\mathrm{pt}, U^{\prime}\right)$;

(b) $h_{0}=\left(z, U^{\prime}, \psi ; s_{z} \circ p^{h}\right)=s_{z} \circ\left(\{z\}, U^{\prime}, \psi ; p^{h}\right) \in \operatorname{Fr}_{m}\left(\mathrm{pt}, U^{\prime}\right)$, where $p^{h}: U^{\prime} \rightarrow \mathrm{pt}=\operatorname{Spec}(k)$ is the structure morphism and $s_{z}: \mathrm{pt} \hookrightarrow U^{\prime}$ is the closed point of $U^{\prime}$.

Lemma 4.12. Let $z \in \mathbb{A}^{m}$ be a k-rational point. Let $Y$ be a $k$-smooth scheme and let $\left(z, U,\left(\varphi_{1}, \varphi_{2}, \ldots, \varphi_{m}\right), g\right) \in \mathrm{Fr}_{m}(\mathrm{pt}, Y)$ be a framed correspondence. Then

$$
\left(z, U,\left(\varphi_{1}, \varphi_{2}, \ldots, \varphi_{m}\right), g\right) \sim\left(z, U,\left(\varphi_{1}, \varphi_{2}, \ldots, \varphi_{m}\right), c_{g(z)}\right),
$$

where $c_{g(z)}=g(z) \circ p: U \stackrel{p}{\rightarrow} \mathrm{pt} \stackrel{g(z)}{\longrightarrow} Y$.

Proof. Let $U^{\prime}, U_{s}^{\prime}, i_{z}$ and $h_{s}$ be as in Corollary 4.11. Let $\pi: U^{\prime} \rightarrow U$ be the canonical morphism. Set $h_{s}^{\prime}=g \circ \pi \circ h_{s} \in \operatorname{Fr}_{m}(\mathrm{pt}, Y)$. We want to check that $h_{1}^{\prime}=(z, U, \varphi, g)$ and $h_{0}^{\prime}=\left(z, U, \varphi, c_{g(z)}\right)$. This will prove our statement. One has,

$$
\begin{aligned}
h_{1}^{\prime}=(g \circ \pi) \circ h_{1}= & (g \circ \pi) \circ\left(z, U^{\prime}, \varphi \circ \pi ; i d_{U^{\prime}}\right)=\left(z, U^{\prime}, \varphi \circ \pi ; g \circ \pi\right)=(z, U, \varphi ; g), \\
h_{0}^{\prime}=(g \circ \pi) \circ h_{0}= & (g \circ \pi) \circ\left(z, U^{\prime}, \varphi \circ \pi ; s_{z} \circ p^{h}\right)=\left(z, U^{\prime}, \varphi \circ \pi ; g \circ \pi \circ s_{z} \circ p^{h}\right)= \\
& =\left(z, U^{\prime}, \varphi \circ \pi ; c_{g(z)} \circ \pi\right)=\left(z, U, \varphi ; c_{g(z)}\right)
\end{aligned}
$$

as required.

Lemma 4.13. Let $Y$ be a $k$-smooth scheme and let $(Z, U, \varphi, g) \in \mathrm{Fr}_{1}(\mathrm{pt}, Y)$ be a framed correspondence. Suppose that $U \subset \mathbb{A}^{1}$ and $\varphi=p(t) \in k[t]$ is a polynomial, where $t$ is the coordinate function on $\mathbb{A}^{1}$. Let $g: U \rightarrow Y$ be a morphism. 
(1) Then for every $a \in k$ we have

$$
(Z, U, p(t), g(t)) \sim\left(m_{a}^{-1}(Z), m_{a}^{-1}(U), p(t-a), g(t-a)\right) \in \mathrm{Fr}_{1}(\mathrm{pt}, Y),
$$

where $m_{a}: \mathbb{A}^{1} \rightarrow \mathbb{A}^{1}$ is given by $m_{a}(t)=t-a$.

(2) If $Z=\left\{x_{0}\right\}$ for some $x_{0} \in k$ and $p(t)=\left(t-x_{0}\right)^{n} r(t)$ with $r(t)$ invertible on $U$, then

$$
(Z, U, p(t), g) \sim\left(\{0\}, \mathbb{A}^{1}, r\left(x_{0}\right) t^{n}, c_{g\left(x_{0}\right)}\right) \in \operatorname{Fr}_{1}(\mathrm{pt}, Y),
$$

where $c_{g\left(x_{0}\right)}: \mathbb{A}^{1} \rightarrow \mathrm{pt} \stackrel{g\left(x_{0}\right)}{\longrightarrow} Y$ is the constant map taking $\mathbb{A}^{1}$ to the point $g\left(x_{0}\right) \in Y$.

Proof. (1) The homotopy is given by

$$
\left(m_{s a}^{-1}(Z), m_{s a}^{-1}(U), p(t-s a), g(t-s a)\right) \in \operatorname{Fr}_{1}\left(\mathbb{A}^{1}, Y\right),
$$

where $s$ is the homotopy parameter and $m_{s a}: \mathbb{A}^{1} \times \mathbb{A}^{1} \rightarrow \mathbb{A}^{1}$ is the morphism $m_{s a}(t)=t-s a$.

(2) Using the preceding statement, we may assume that $x_{0}=0$. Consider a polynomial

$$
h(s, t)=s r(t) t^{n}+(1-s) r(0) t^{n} \in k[s, t] .
$$

If $r_{1}(t)$ is such that $r(t)=r(0)+t \cdot r_{1}(t)$, then one has $h(s, t)=t^{n} \cdot\left(r(0)+t \cdot r_{1}(t) \cdot s\right)$. If $S$ is the vanishing locus of $r(0)+t \cdot r_{1}(t) \cdot s$, then $S \cap \mathbb{A}^{1} \times 0=\emptyset$. Hence for the zero locus $Z(h)$ of $h$ one has $Z(h)=\left(\mathbb{A}^{1} \times 0\right) \sqcup S$. The framed correspondence

$$
\left(\mathbb{A}^{1} \times\{0\},\left(\mathbb{A}^{1} \times U\right) \backslash S, s r(t) t^{n}+(1-s) r(0) t^{n}, g \circ p r_{U}\right) \in \operatorname{Fr}_{1}\left(\mathbb{A}^{1}, Y\right)
$$

yields the relation $\left(\{0\}, U, r(t) t^{n}, g\right) \sim\left(\{0\}, U, r(0) t^{n}, g\right)$ in $\operatorname{Fr}_{1}(\mathrm{pt}, Y)$. Lemma 4.12 shows that

$$
\left(\{0\}, U, r(0) t^{n}, g\right) \sim\left(\{0\}, U, r(0) t^{n}, g(0)\right)=\left(\{0\}, \mathbb{A}^{1}, r(0) t^{n}, g(0)\right) \in \operatorname{Fr}_{1}(\mathrm{pt}, Y)
$$

and our lemma follows.

Lemma 4.14. Let $a \in k^{\times}$. Let $p(t), q(t) \in k[t]$ be two polynomials of degree $n$ with the leading coefficient a. Let $\left(Z(p), \mathbb{A}^{1}, p(t), c\right) \in \operatorname{Fr}_{1}(\mathrm{pt}, \mathrm{pt}),\left(Z(q), \mathbb{A}^{1}, q(t), c\right) \in \mathrm{Fr}_{1}(\mathrm{pt}, \mathrm{pt})$ be two framed correspondences. Here $c: \mathbb{A}^{1} \rightarrow \mathrm{pt}$ is the structure morphism. Then

$$
\left(Z(p), \mathbb{A}^{1}, p(t), c\right) \sim\left(Z(q), \mathbb{A}^{1}, q(t), c\right) \in \mathrm{Fr}_{1}(\mathrm{pt}, \mathrm{pt}) .
$$

Proof. As a polinomial in $t$ the leading coefficient of the polinomial $p(t)+s(q(t)-p(t))$ is $a \in k^{\times}$. Hence the $k[s]$-module $k[s, t] /(p(t)+s(q(t)-p(t)))$ is a free module rank $n$. Let $Z_{s} \subset \mathbb{A}^{1} \times \mathbb{A}^{1}$ be the vanishing locus of $p(t)+s(q(t)-p(t))$. The desired homotopy is given by the framed correspondence

$$
\left(Z_{s}, \mathbb{A}^{1} \times \mathbb{A}^{1}, p(t)+s(q(t)-p(t)), c^{\prime}\right),
$$

where $s$ is the homotopy parameter and $c^{\prime}: \mathbb{A}^{1} \times \mathbb{A}^{1} \rightarrow$ pt is the canonical projection.

\section{HOMOTOPIES FOR SWAPPING COORDINATES OF $\mathbb{G}_{m} \times \mathbb{G}_{m}$}

In this section we follow notation of Section 2. Denote by $\varepsilon=\left(\{0\}, \mathbb{A}^{1},-t, c\right) \in \operatorname{Fr}_{1}(\mathrm{pt}$, pt), where $c: \mathbb{A}^{1} \rightarrow \mathrm{pt}$ is the canonical projection. We work in this Section with the elements $\Sigma^{n} \in$ $\mathbb{Z F}_{n}(\mathrm{pt}, \mathrm{pt})$ as in Definitition 2.3.

Proposition 5.1. Let $Y$ be a $k$-smooth scheme. Then the canonical homomorphism

$$
H_{0}\left(\mathbb{Z} \mathrm{F}\left(\Delta^{\bullet} \times \mathbb{G}_{m} \times \mathbb{G}_{m}, Y\right)\right) \rightarrow H_{0}\left(\mathbb{Z} \mathrm{F}\left(\Delta_{\text {Spec } k(t, u)}^{\bullet}, Y\right)\right)
$$

is injective. 
Proof. By [GP2, 3.15(1)] the canonical homomorhisms

$$
H_{0}\left(\mathbb{Z F}\left(\Delta^{\bullet} \times \mathbb{G}_{m} \times \mathbb{G}_{m}, Y\right)\right) \rightarrow H_{0}\left(\mathbb{Z F}\left(\Delta^{\bullet} \times \mathbb{G}_{m, k(u)}, Y\right)\right)
$$

and

$$
H_{0}\left(\mathbb{Z F}\left(\Delta^{\bullet} \times \mathbb{G}_{m, k(u)}, Y\right)\right) \rightarrow H_{0}\left(\mathbb{Z F}\left(\Delta_{\operatorname{Spec} k(t, u)}^{\bullet}, Y\right)\right)
$$

are injective, hence the lemma.

Let $Y$ be a $k$-smooth variety and $F / k$ be a field extension. There is a map of pointed sets

$$
\text { adj: } \operatorname{Fr}_{n}(\operatorname{Spec}(F), Y) \rightarrow \operatorname{Fr}_{n}^{F}\left(\operatorname{Spec}(F), Y_{F}\right)
$$

given by the assignment $(Z, W, \varphi, g) \mapsto\left(Z, W, \varphi^{F}, g^{F}\right)$. Here for a $k$-morphism $g: W \rightarrow Y$ we write $g^{F}$ to denote the $F$-morphism $\left(g, p r_{\operatorname{Spec}(F)}\right): W \rightarrow Y_{F}$ and $p r_{\operatorname{Spec}(F)}: W \rightarrow \operatorname{Spec}(F)$ is the structure morphism. In particular, for $Y=\mathbb{A}^{n}$ and $\varphi: W \rightarrow \mathbb{A}_{k}^{n}$ we write $\varphi^{F}$ for $\left(\varphi, p r_{\operatorname{Spec}(F)}\right): W \rightarrow \mathbb{A}_{F}^{n}$. It is easy to see that the map $a d j$ is a bijection. Moreover, it induces bijections

$$
\text { adj }: \mathbb{Z F}_{n}(\operatorname{Spec}(F), Y) \rightarrow \mathbb{Z F}_{n}^{F}\left(\operatorname{Spec}(F), Y_{F}\right) \text { and } \mathbb{Z} \mathrm{F}(\operatorname{Spec}(F), Y) \rightarrow \mathbb{Z F}^{F}\left(\operatorname{Spec}(F), Y_{F}\right) .
$$

Lemma 5.2. Let $F / k$ be a field extension, choose $x, y \in F^{\times}$such that $x \neq y^{ \pm 1}$ and let $u_{1}, u_{2}$ be coordinates on $\mathbb{G}_{m} \times \mathbb{G}_{m}$. Consider morphisms $f, g$ : Spec $F \rightarrow \mathbb{G}_{m} \times \mathbb{G}_{m}$ given by $u_{1} \mapsto x, u_{2} \mapsto y$ and $u_{1} \mapsto y, u_{2} \mapsto x$ respectively. Then for $p=\left(\mathrm{id}-e_{1}\right) \otimes\left(\mathrm{id}-e_{1}\right)$ we have

$$
p \circ(f \otimes \Sigma) \sim p \circ(g \otimes(-\varepsilon))
$$

in $\mathbb{Z F}\left(\operatorname{Spec} F, \mathbb{G}_{m} \times \mathbb{G}_{m}\right)$.

Proof. The above adjunction isomorphism

$$
\text { adj: } \mathbb{Z} \mathrm{F}\left(\operatorname{Spec} F, \mathbb{G}_{m} \times \mathbb{G}_{m}\right) \cong \mathbb{Z} \mathrm{F}^{F}\left(\operatorname{Spec} F, \mathbb{G}_{m, F} \times \mathbb{G}_{m, F}\right)
$$

implies it is sufficient to verify the case $F=k$. So we have morphisms $f, g: \mathrm{pt} \rightarrow \mathbb{G}_{m} \times \mathbb{G}_{m}$, $\mathrm{pt} \mapsto(x, y)$ and $\mathrm{pt} \mapsto(y, x)$ respectively. Taking suspensions, we obtain framed correspondences

$$
\left(\{0\}, \mathbb{A}^{1}, t, c_{(x, y)}\right),\left(\{0\}, \mathbb{A}^{1}, t, c_{(y, x)}\right) \in \mathrm{Fr}_{1}\left(\mathrm{pt}, \mathbb{G}_{m} \times \mathbb{G}_{m}\right),
$$

where $c_{(x, y)}$ and $c_{(y, x)}$ are morphisms on $\mathbb{A}^{1}$ sending it to the points $(x, y)$ and $(y, x)$ respectively.

Consider $h(s, t)=\frac{1}{x-y}\left(t^{2}-(s(x+y)+(1-s)(x y+1)) t+x y\right) \in k\left[s, t, t^{-1}\right]=k\left[\mathbb{A}^{1} \times \mathbb{G}_{m}\right]$ and a framed correspondence

$$
H_{s}:=\left(Z(h), \mathbb{A}^{1} \times \mathbb{G}_{m}, h(s, t),\left(t, x y t^{-1}\right) \circ \operatorname{pr}_{\mathbb{G}_{m}}\right) \in \operatorname{Fr}_{1}\left(\mathbb{A}^{1}, \mathbb{G}_{m} \times \mathbb{G}_{m}\right) .
$$

We have $h(0, t)=\frac{1}{x-y}(t-x y)(t-1)$ and $h(1, t)=\frac{1}{x-y}(t-x)(t-y)$. Using the additivity property for supports in $\mathbb{Z F}_{1}\left(\mathrm{pt}, \mathbb{G}_{m} \times \mathbb{G}_{m}\right)$ (see Definition 2.4) and Lemma 4.13 we will check below that

$$
\left(\{0\}, \mathbb{A}^{1}, t, c_{(x, y)}\right)+\left(\{0\}, \mathbb{A}^{1},-t, c_{(y, x)}\right) \sim\left(\{0\}, \mathbb{A}^{1}, \frac{1-x y}{x-y} t, c_{(1, x y)}\right)+\left(\{0\}, \mathbb{A}^{1}, \frac{x y-1}{x-y} t, c_{(x y, 1)}\right)
$$

in $\mathbb{Z} \mathrm{F}_{1}\left(\mathrm{pt}, \mathbb{G}_{m} \times \mathbb{G}_{m}\right)$. The composition with the idempotent $p$ annihilates all extra summands and proves the lemma.

In order to prove the relation (5), consider the framed correspondence (4) in $\mathbb{Z} F_{1}\left(\mathbb{A}^{1}, \mathbb{G}_{m} \times \mathbb{G}_{m}\right)$. Observe that in $\mathbb{Z} \mathrm{F}_{1}\left(\mathrm{pt}, \mathbb{G}_{m} \times \mathbb{G}_{m}\right)$

$$
\begin{aligned}
& H_{1}=\left(Z\left(h(t, 1), \mathbb{G}_{m}, h(1, t),\left(t, x y t^{-1}\right)\right)=\right. \\
= & \left(\{x\}, \mathbb{G}_{m}-\{y\}, \frac{1}{x-y}(t-x)(t-y),\left(t, x y t^{-1}\right)\right)+\left(\{y\}, \mathbb{G}_{m}-\{x\}, \frac{1}{x-y}(t-x)(t-y),\left(t, x y t^{-1}\right)\right) .
\end{aligned}
$$


By Lemma 4.13 one has in $\mathbb{Z F}_{1}\left(\mathrm{pt}, \mathbb{G}_{m} \times \mathbb{G}_{m}\right)$

$$
\begin{gathered}
\left(\{x\}, \mathbb{G}_{m}-\{y\}, \frac{1}{x-y}(t-x)(t-y),\left(t, x y t^{-1}\right)\right) \sim\left(\{0\}, \mathbb{A}^{1}, \frac{x-y}{x-y} t, c_{(x, y)}\right)=\left(\{0\}, \mathbb{A}^{1}, t, c_{(x, y)}\right), \\
\left(\{y\}, \mathbb{G}_{m}-\{x\}, \frac{1}{x-y}(t-x)(t-y),\left(t, x y t^{-1}\right)\right) \sim\left(\{0\}, \mathbb{A}^{1}, \frac{y-x}{x-y} t, c_{(x, y)}\right)=\left(\{0\}, \mathbb{A}^{1},-t, c_{(x, y)}\right) .
\end{gathered}
$$

Thus $H_{1} \sim\left(\{0\}, \mathbb{A}^{1}, t, c_{(x, y)}\right)+\left(\{0\}, \mathbb{A}^{1},-t, c_{(y, x)}\right)$ in $\mathbb{Z F}_{1}\left(\mathrm{pt}, \mathbb{G}_{m} \times \mathbb{G}_{m}\right)$. Similar computations show that $H_{0} \sim\left(\{0\}, \mathbb{A}^{1}, \frac{1-x y}{x-y} t, c_{(1, x y)}\right)+\left(\{0\}, \mathbb{A}^{1}, \frac{x y-1}{x-y} t, c_{(x y, 1)}\right)$ in $\mathbb{Z F}_{1}\left(\mathrm{pt}, \mathbb{G}_{m} \times \mathbb{G}_{m}\right)$. The equality (5) is proved. Since the right hand side of the equality (5) is annihilated by the idempotent $p$, our lemma follows.

Proposition 5.3. Let $\tau: \mathbb{G}_{m} \times \mathbb{G}_{m} \rightarrow \mathbb{G}_{m} \times \mathbb{G}_{m}$ be the permutation of coordinates morphism. Denote $p=\left(\mathrm{id}-e_{1}\right) \otimes\left(\mathrm{id}-e_{1}\right)$. Then $p \circ\left(\operatorname{id}_{\mathbb{G}_{m} \times \mathbb{G}_{m}} \otimes(-\varepsilon)\right) \sim p \circ(\tau \otimes \Sigma)$ in $\mathbb{Z} \mathrm{F}\left(\mathbb{G}_{m} \times \mathbb{G}_{m}, \mathbb{G}_{m} \times \mathbb{G}_{m}\right)$.

Proof. Note that $\tau \otimes(-\varepsilon)=\left(\mathrm{id}_{\mathbb{G}_{m} \times \mathbb{G}_{m}} \otimes(-\varepsilon)\right) \circ \tau$. Hence

$$
\left.p \circ(\tau \otimes(-\varepsilon)) \circ \tau=p \circ\left(\operatorname{id}_{\mathbb{G}_{m} \times \mathbb{G}_{m}} \otimes(-\varepsilon)\right) \in \mathbb{Z F}_{1}\left(\mathbb{G}_{m} \times \mathbb{G}_{m}, \mathbb{G}_{m} \times \mathbb{G}_{m}\right)\right) .
$$

Similarly, $p \circ\left(\operatorname{id}_{\mathbb{G}_{m} \times \mathbb{G}_{m}} \otimes \Sigma\right) \circ \tau=p \circ(\tau \otimes \Sigma)$ in $\left.\mathbb{Z} F_{1}\left(\mathbb{G}_{m} \times \mathbb{G}_{m}, \mathbb{G}_{m} \times \mathbb{G}_{m}\right)\right)$. It remains to check that

$$
p \circ\left(\operatorname{id}_{\mathbb{G}_{m} \times \mathbb{G}_{m}} \otimes \Sigma\right)=p \circ(\tau \otimes(-\varepsilon))
$$

in $H_{0}\left(\mathbb{Z} \mathrm{F}\left(\Delta^{\bullet} \times \mathbb{G}_{m} \times \mathbb{G}_{m}, \mathbb{G}_{m} \times \mathbb{G}_{m}\right)\right)$.

Let $u_{1}$ and $u_{2}$ be coordinate functions on $\mathbb{G}_{m} \times \mathbb{G}_{m}$. Let $f: \operatorname{Spec} k\left(u_{1}, u_{2}\right) \rightarrow \operatorname{Spec} k\left[u_{1}, u_{2}\right]$ be the canonical embedding and $g: \operatorname{Spec} k\left(u_{1}, u_{2}\right) \rightarrow \operatorname{Spec} k\left[u_{1}, u_{2}\right]$ be given by $g^{*}\left(u_{1}\right)=u_{2}, g^{*}\left(u_{2}\right)=$ $u_{1}$. By Proposition 5.2 we know that $p \circ(f \otimes \Sigma) \sim p \circ(g \otimes(-\varepsilon))$ in $H_{0}\left(\mathbb{Z F}\left(\Delta_{k\left(u_{1}, u_{2}\right)}^{\bullet}, \mathbb{G}_{m} \times \mathbb{G}_{m}\right)\right)$. Proposition 5.1 yields the desired equality (6) in $H_{0}\left(\mathbb{Z F}\left(\Delta^{\bullet} \times \mathbb{G}_{m} \times \mathbb{G}_{m}, \mathbb{G}_{m} \times \mathbb{G}_{m}\right)\right)$.

Recall that $\Sigma=\left(\{0\}, \mathbb{A}^{1}, t\right) \in \mathbb{Z F}_{1}(\mathrm{pt}, \mathrm{pt})$. For every $k>0$ we write $\Sigma^{k}$ to denote $\Sigma \otimes \cdots \cdot \otimes \Sigma \in$ $\mathbb{Z} \mathrm{F}_{k}(\mathrm{pt}, \mathrm{pt})$.

Let $\tau: \mathbb{G}_{m} \times \mathbb{G}_{m} \rightarrow \mathbb{G}_{m} \times \mathbb{G}_{m}$ be the permutation of coordinates morphism. For each even integer $m \geqslant 0$ and each integer $n \geqslant 0$ consider two presheaf morphisms

$\left(-\otimes \Sigma^{2 n}\right): \mathbb{Z}_{m}\left(-\times X \times \mathbb{G}_{m} \times \mathbb{G}_{m}, Y \times \mathbb{G}_{m} \times \mathbb{G}_{m}\right) \rightarrow \mathbb{Z F}_{m+2 n}\left(-\times X \times \mathbb{G}_{m} \times \mathbb{G}_{m}, Y \times \mathbb{G}_{m} \times \mathbb{G}_{m}\right)$, $\left(-\otimes \Sigma^{2 n}\right) \circ s w: \mathbb{Z F}_{m}\left(-\times X \times \mathbb{G}_{m} \times \mathbb{G}_{m}, Y \times \mathbb{G}_{m} \times \mathbb{G}_{m}\right) \rightarrow \mathbb{Z F}_{m+2 n}\left(-\times X \times \mathbb{G}_{m} \times \mathbb{G}_{m}, Y \times \mathbb{G}_{m} \times \mathbb{G}_{m}\right)$, where $s w(a)=\left(\operatorname{id}_{Y} \times \tau\right) \circ a \circ\left(\operatorname{id}_{X} \times \tau\right)$.

Lemma 5.4. Let $X, Y$ be $k$-smooth schemes. Given an even integer $m \geqslant 0$, there exists a large enough $n$ and a homotopy

$$
H: \mathbb{Z F}_{m}\left(-\times X \times \mathbb{G}_{m} \times \mathbb{G}_{m}, Y \times \mathbb{G}_{m} \times \mathbb{G}_{m}\right) \rightarrow \mathbb{Z F}_{m+2 n}\left(-\times X \times \mathbb{G}_{m} \times \mathbb{G}_{m} \times \mathbb{A}^{1}, Y \times \mathbb{G}_{m} \times \mathbb{G}_{m}\right)
$$

such that for any $a \in \mathbb{Z F}_{m}\left(W \times X \wedge\left(\mathbb{G}_{m}, 1\right) \wedge\left(\mathbb{G}_{m}, 1\right), Y \wedge\left(\mathbb{G}_{m}, 1\right) \wedge\left(\mathbb{G}_{m}, 1\right)\right)$ one has

$$
a \otimes \Sigma^{2 n}=H_{0}(a) \text { and } H_{1}(a)=\Sigma^{2 n}\left(\left[\left(\operatorname{id}_{Y} \times \tau\right) \circ a \circ\left(\operatorname{id}_{X} \times \tau\right)\right]\right) \text {. }
$$

And both $H_{0}(a)$ and $H_{1}(a)$ are in $\mathbb{Z F}_{m+2 n}\left(W \times X \wedge\left(\mathbb{G}_{m}, 1\right) \wedge\left(\mathbb{G}_{m}, 1\right), Y \wedge\left(\mathbb{G}_{m}, 1\right) \wedge\left(\mathbb{G}_{m}, 1\right)\right)$.

Proof. It follows from Proposition 5.3 that there exists a large enough integer $n$ and a homotopy $\Psi \in \mathbb{Z F}_{n}\left(\mathbb{G}_{m} \times \mathbb{G}_{m} \times \mathbb{A}^{1}, \mathbb{G}_{m} \times \mathbb{G}_{m}\right)$ such that $i_{0}^{*}(\Psi)=p \circ\left(-\varepsilon \otimes\left(\Sigma^{n-1} \operatorname{id}_{\mathbb{G}_{m} \times \mathbb{G}_{m}}\right)\right)$ and $i_{1}^{*}(\Psi)=$ $p \circ \Sigma^{n} \tau$, where $p=\left(\mathrm{id}-e_{1}\right) \otimes\left(\mathrm{id}-e_{1}\right)$. 
Given any element $a \in \mathbb{Z F}_{m}\left(W \times X \times \mathbb{G}_{m} \times \mathbb{G}_{m}, Y \times \mathbb{G}_{m} \times \mathbb{G}_{m}\right)$, set

$$
\begin{aligned}
& H^{\prime}(a)=\left(i d_{Y} \times \Psi\right) \circ\left(a \times i d_{\mathbb{A}^{1}}\right) \circ\left(i d_{W \times X} \times \Psi \times i d_{\mathbb{A}^{1}}\right) \circ\left(i d_{W \times X \times \mathbb{G}_{m} \times \mathbb{G}_{m}} \times \Delta\right) \in \\
& \in \mathbb{Z F}_{m+2 n}\left(W \times X \times \mathbb{G}_{m} \times \mathbb{G}_{m} \times \mathbb{A}^{1}, Y \times \mathbb{G}_{m} \times \mathbb{G}_{m}\right),
\end{aligned}
$$

where $\Delta: \mathbb{A}^{1} \rightarrow \mathbb{A}^{1} \times \mathbb{A}^{1}$ is the diagonal morphism. Then for any element $a \in \mathbb{Z} \mathrm{F}_{m}(W \times X \wedge$ $\left.\left(\mathbb{G}_{m}, 1\right) \wedge\left(\mathbb{G}_{m}, 1\right), Y \wedge\left(\mathbb{G}_{m}, 1\right) \wedge\left(\mathbb{G}_{m}, 1\right)\right)$ one has

$$
H^{\prime}(a)_{0}=\left[i d_{Y} \times \Sigma^{n-1}(-\varepsilon)\right] \circ a \circ\left[i d_{X} \times \Sigma^{n-1}(-\varepsilon)\right] \text { and } H^{\prime}(a)_{1}=\left[\operatorname{id}_{Y} \times \Sigma^{n}(\tau)\right] \circ a \circ\left[\operatorname{id}_{X} \times \Sigma^{n}(\tau)\right] \text {. }
$$

It is easy to see that there are matrices $A, B \in S L_{m+2 n}(k)$ such that for any element $a$ in $\mathbb{Z} \mathrm{F}_{m}(W \times$ $\left.X \wedge\left(\mathbb{G}_{m}, 1\right) \wedge\left(\mathbb{G}_{m}, 1\right), Y \wedge\left(\mathbb{G}_{m}, 1\right) \wedge\left(\mathbb{G}_{m}, 1\right)\right)$ one has

$$
\begin{gathered}
\varphi_{A}\left(\left[i d_{Y} \times \Sigma^{n-1}(-\varepsilon)\right] \circ a \circ\left[i d_{X} \times \Sigma^{n-1}(-\varepsilon)\right]\right)=a \otimes \Sigma^{2 n}=\Sigma^{2 n}(a), \\
\varphi_{B}\left(\left[\operatorname{id}_{Y} \times \Sigma^{n}(\tau)\right] \circ a \circ\left[\operatorname{id}_{X} \times \Sigma^{n}(\tau)\right]\right)=\left(\left[\operatorname{id}_{Y} \times \tau\right] \circ a \circ\left[\operatorname{id}_{X} \times \tau\right)\right) \otimes \Sigma^{2 n}=\Sigma^{2 n}\left(\left[\operatorname{id}_{Y} \times \tau\right] \circ a \circ\left[\operatorname{id}_{X} \times \tau\right]\right) .
\end{gathered}
$$

Choose matrices $A_{s}, B_{s} \in S L_{m+2 n}(k[s])$ such that $A_{0}=i d, A_{1}=A, B_{0}=i d, B_{1}=B$. Then for the matrix $C_{s}=B_{s} \circ A_{1-s} \in S L_{m+2 n}(k[s])$ one has $C_{0}=A, C_{1}=B$. Set $H=\varphi_{C_{s}} \circ H^{\prime}$. Then for the chosen element $a \in \mathbb{Z F}_{m}\left(W \times X \wedge\left(\mathbb{G}_{m}, 1\right) \wedge\left(\mathbb{G}_{m}, 1\right), Y \wedge\left(\mathbb{G}_{m}, 1\right) \wedge\left(\mathbb{G}_{m}, 1\right)\right)$, one has

$$
H_{0}(a)=\varphi_{A}\left(H^{\prime}(a)_{0}\right)=\Sigma^{2 n}(a) \text { and } H_{1}(a)=\varphi_{B}\left(H^{\prime}(a)_{1}\right)=\Sigma^{2 n}\left(\left[\operatorname{id}_{Y} \times \tau\right] \circ a \circ\left[\operatorname{id}_{X} \times \tau\right)\right),
$$

as was to be proved.

\section{THE INVERSE MORPHISM}

The main aim of this section is to define for any integers $n, m \geqslant 0$ a subpresheaf $\mathbb{Z} \mathrm{F}_{m}^{(n)}(-\times$ $\left.\mathbb{G}_{m}, Y \times \mathbb{G}_{m}\right)$ of the presheaf $\mathbb{Z F}_{m}\left(-\times \mathbb{G}_{m}, Y \times \mathbb{G}_{m}\right)$ and define a morphism of abelian presheaves

$$
\rho_{n}: \mathbb{Z} \mathrm{F}_{m}^{(n)}\left(-\times \mathbb{G}_{m}, Y \times \mathbb{G}_{m}\right) \rightarrow \mathbb{Z}_{m}(-, Y) .
$$

We also prove certain properties of morphisms $\rho_{n}$ and of presheaves $\mathbb{Z} \mathrm{F}_{m}^{(n)}\left(-\times \mathbb{G}_{m}, Y \times \mathbb{G}_{m}\right)$ which are used in the proof of the Linear Cancelation Theorem (Theorem C).

We begin with some general remarks. Let $X$ and $Y$ be $k$-smooth schemes. Consider a framed correspondence

$$
a=\left(Z, U,\left(\varphi_{1}, \varphi_{2}, \ldots, \varphi_{m}\right), g\right) \in \mathrm{Fr}_{m}\left(X \times \mathbb{G}_{m}, Y \times \mathbb{G}_{m}\right) .
$$

Let $\left(U, p: U \rightarrow \mathbb{A}^{m} \times\left(X \times \mathbb{G}_{m}\right), s: Z \rightarrow U\right)$ be the étale neighborhood of $Z$ in $\mathbb{A}^{m} \times\left(X \times \mathbb{G}_{m}\right)$ from the definition of the framed correspondence $a$. Let $t$ be the invertible function on $X \times \mathbb{G}_{m}$ corresponding to the projection on $\mathbb{G}_{m}$ and $u$ be invertible function on $Y \times \mathbb{G}_{m}$ corresponding to the projection on $\mathbb{G}_{m}$. Let $f_{2}=g^{*}(u)$ and $f_{1}=p_{X \times \mathbb{G}_{m}}^{*}(t)$ be two invertible functions on $U$, where $p_{X \times \mathbb{G}_{m}}=p r_{X \times \mathbb{G}_{m}} \circ p: U \rightarrow X \times \mathbb{G}_{m}$. Set $g=\left(g_{1}, g_{2}\right)$, where $g_{1}=p r_{Y} \circ g$ and $g_{2}=p r_{\mathbb{G}_{m}} \circ g$.

Since $Z$ is finite over $X \times \mathbb{G}_{m}$, the $\mathscr{O}_{X \times \mathbb{G}_{m} \times Y \times \mathbb{G}_{m}}$-sheaf $P_{a}:=\mathscr{O}_{U} /\left(\varphi_{1}, \varphi_{2}, \ldots, \varphi_{m}\right)$ is finite over $X \times \mathbb{G}_{m}$. Since the sheaf $P_{a}$ is finite over $X \times \mathbb{G}_{m}$, it is flat over $X \times \mathbb{G}_{m}$ by [OP, Lemma 7.3].

Let $Z_{n}^{+}$be the closed subset of $Z$ defined by the equation $\left.\left(f_{1}^{n+1}-1\right)\right|_{Z}=0$. Let $Z_{n}^{-}$be the closed subset of $Z$ defined by the equation $\left.\left(f_{1}^{n+1}-f_{2}\right)\right|_{Z}=0$. Note that $Z_{n}^{+}$is finite over $X$ if and only if $\mathscr{O}_{U} /\left(f_{1}^{n+1}-1, \varphi_{1}, \varphi_{2}, \ldots, \varphi_{m}\right)$ is finite over $X$. By [S, 4.1] the latter $\mathscr{O}_{X}$-module is always finite and even flat. Note that $Z_{n}^{-}$is finite over $X$ if and only if $\mathscr{O}_{U} /\left(f_{1}^{n+1}-f_{2}, \varphi_{1}, \varphi_{2}, \ldots, \varphi_{m}\right)$ is finite over $X$. We have mentioned above that the $\mathscr{O}_{X \times \mathbb{G}_{m}}$-module $P_{a}=\mathscr{O}_{U} /\left(\varphi_{1}, \varphi_{2}, \ldots, \varphi_{m}\right)$ is finite and flat over $\mathscr{O}_{X \times \mathbb{G}_{m}}$. Thus by [S, 4.1.b] there exists an integer $N$ such that for any $n \geqslant N$ the $\mathscr{O}_{X}$-module $\mathscr{O}_{U} /\left(f_{1}^{n+1}-f_{2}, \varphi_{1}, \varphi_{2}, \ldots, \varphi_{m}\right)$ is finite and even flat over $X$. In particular, $Z_{n}^{-}$is finite over $X$ for any $n \geqslant N$. 
The following definition is inspired by [S, Section 4].

Definition 6.1. Let $X$ and $Y$ be $k$-smooth schemes. Consider a framed correspondence $a=$ $\left(Z, U,\left(\varphi_{1}, \varphi_{2}, \ldots, \varphi_{m}\right), g\right) \in \operatorname{Fr}_{m}\left(X \times \mathbb{G}_{m}, Y \times \mathbb{G}_{m}\right)$. Set

$$
\rho_{n, f r}^{+}(a):=\left(Z_{n}^{+}, U,\left(f_{1}^{n+1}-1, \varphi_{1}, \varphi_{2}, \ldots, \varphi_{m}\right), g_{1}\right)
$$

and

$$
\rho_{n, f r}^{-}(a):=\left(Z_{n}^{-}, U,\left(f_{1}^{n+1}-f_{2}, \varphi_{1}, \varphi_{2}, \ldots, \varphi_{m}\right), g_{1}\right) .
$$

As we have mentioned above, $Z_{n}^{+}$is finite over $X$ for all $n \geqslant 0$, hence $\rho_{n, f r}^{+}(a) \in \mathbb{Z F}_{m+1}(X, Y)$. We say that $\rho_{n, f r}^{-}(a)$ is defined if $Z_{n}^{-}$is finite over $X$, which is equivalent to saying that the $\mathscr{O}_{X}$-module $P_{a} /\left(f_{1}^{n+1}-f_{2}\right) P_{a}$ is finite and flat over $X$. If $\rho_{n, f r}^{-}(a)$ is defined, then we set

$$
\rho_{n, f r}(a)=\rho_{n, f r}^{+}(a)-\rho_{n, f r}^{-}(a) \in \mathbb{Z F}_{m+1}(X, Y)
$$

and say that $\rho_{n, f r}(a)$ is defined.

Given integers $m, n \geqslant 0$, denote by $\operatorname{Fr}_{m}^{(n)}\left(X \times \mathbb{G}_{m}, Y \times \mathbb{G}_{m}\right)$ the subset of those framed correspondences $a \in \operatorname{Fr}_{m}\left(X \times \mathbb{G}_{m}, Y \times \mathbb{G}_{m}\right)$ for which the $\mathscr{O}_{X}$-module $P_{a} /\left(f_{1}^{n+1}-f_{2}\right) P_{a}$ is finite over $X$ (that is $\rho_{n, f r}(a)$ is defined). It follows from [S, 4.4] that the assignment $X^{\prime} \mapsto \operatorname{Fr}_{m}^{(n)}\left(X^{\prime} \times \mathbb{G}_{m}, Y \times \mathbb{G}_{m}\right)$ is a subpresheaf of $\operatorname{Fr}_{m}\left(-\times \mathbb{G}_{m}, Y \times \mathbb{G}_{m}\right)$.

Definition 6.2. Define a presheaf of abelian groups $\mathbb{Z F}_{m}^{(n)}\left(-\times \mathbb{G}_{m}, Y \times \mathbb{G}_{m}\right)$ as follows. Its sections on $X$ is the abelian group $\mathbb{Z}\left[\operatorname{Fr}_{m}^{(n)}\left(X \times \mathbb{G}_{m}, Y \times \mathbb{G}_{m}\right)\right]$ modulo a subgroup generated by all elements of the form

$$
\left(Z_{1} \sqcup Z_{2}, U_{1} \sqcup U_{2}, \varphi_{1} \sqcup \varphi_{2}, g_{1} \sqcup g_{2}\right)-\left(Z_{1}, U_{1}, \varphi_{1}, g_{1}\right)-\left(Z_{2}, U_{2}, \varphi_{2}, g_{2}\right) .
$$

It is straightforward to check that $\mathbb{Z F}_{m}^{(n)}\left(X \times \mathbb{G}_{m}, Y \times \mathbb{G}_{m}\right)$ is a free abelian group with a free basis consisting of the elements of the form $a=(Z, U, \varphi, g)$, where $Z$ is connected and the $\mathscr{O}_{X}$-module $P_{a} /\left(f_{1}^{n+1}-f_{2}\right) P_{a}$ is finite and flat over $X$. Moreover, the group $\mathbb{Z} \mathrm{F}_{m}^{(n)}\left(X \times \mathbb{G}_{m}, Y \times \mathbb{G}_{m}\right)$ is a subgroup of the group $\mathbb{Z F}_{m}\left(X \times \mathbb{G}_{m}, Y \times \mathbb{G}_{m}\right)$, and $\mathbb{Z F}_{m}^{(n)}\left(-\times \mathbb{G}_{m}, Y \times \mathbb{G}_{m}\right)$ is a subpresheaf of the presheaf $\mathbb{Z} \mathrm{F}_{m}\left(-\times \mathbb{G}_{m}, Y \times \mathbb{G}_{m}\right)$.

It follows from [S, 4.4] that for any morphism $f: X^{\prime} \rightarrow X$ of smooth varieties the following diagram is commutative

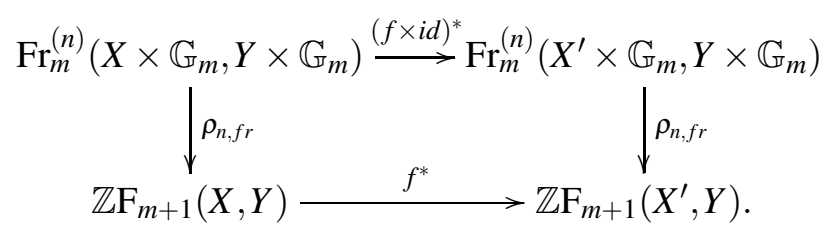

We see that $\rho_{n, f r}: \operatorname{Fr}_{m}^{(n)}\left(-\times \mathbb{G}_{m}, Y \times \mathbb{G}_{m}\right) \rightarrow \mathbb{Z F}_{m+1}(-, Y)$ is a morphism of pointed presheaves. We can extend it to get a morphism of presheaves of abelian groups $\mathbb{Z}\left[\operatorname{Fr}_{m}^{(n)}\left(-\times \mathbb{G}_{m}, Y \times \mathbb{G}_{m}\right)\right] \rightarrow$ $\mathbb{Z} \mathrm{F}_{m+1}(-, Y)$. This morphism annihilates the elements of the form

$$
\left(Z_{1} \sqcup Z_{2}, U_{1} \sqcup U_{2}, \varphi_{1} \sqcup \varphi_{2}, g_{1} \sqcup g_{2}\right)-\left(Z_{1}, U_{1}, \varphi_{1}, g_{1}\right)-\left(Z_{2}, U_{2}, \varphi_{2}, g_{2}\right) \text {. }
$$

Definition 6.3. The above arguments show that the presheaf morphism $\rho_{n, f r}$ induces a unique presheaf of abelian groups morphism

$$
\rho_{n}: \mathbb{Z F}_{m}^{(n)}\left(-\times \mathbb{G}_{m}, Y \times \mathbb{G}_{m}\right) \rightarrow \mathbb{Z F}_{m+1}(-, Y)
$$


such that for any $a \in F_{m}^{(n)}\left(X \times \mathbb{G}_{m}, Y \times \mathbb{G}_{m}\right)$ one has $\rho_{n}(a)=\rho_{n, f r}(a)$. We also call $\rho_{n}$ the inverse morphism.

Lemma 6.4. The following relations are true:

$$
\begin{aligned}
\operatorname{Fr}_{m}\left(-\times \mathbb{G}_{m}, Y \times \mathbb{G}_{m}\right) & =\operatorname{colim}_{n} \mathrm{Fr}_{m}^{(n)}\left(-\times \mathbb{G}_{m}, Y \times \mathbb{G}_{m}\right), \\
\mathbb{Z F}_{m}\left(-\times \mathbb{G}_{m}, Y \times \mathbb{G}_{m}\right) & =\operatorname{colim}_{n} \mathbb{Z F}_{m}^{(n)}\left(-\times \mathbb{G}_{m}, Y \times \mathbb{G}_{m}\right) .
\end{aligned}
$$

This lemma follows from the following

Proposition 6.5. ([S, 4.1]) For any framed correspondence a $\in \operatorname{Fr}_{m}\left(X \times \mathbb{G}_{m}, Y \times \mathbb{G}_{m}\right)$ one has:

(a) for any $n \geqslant 0$, the sheaf $P_{a} /\left(f_{1}^{n+1}-1\right) P_{a}$ is finite and flat over $X$;

(b) there exists an integer $N$ such that, for any $n \geqslant N$, the sheaf $P_{a} /\left(f_{1}^{n+1}-f_{2}\right) P_{a}$ is finite and flat over X.

We shall need the following obvious property of $\rho_{n}$.

Lemma 6.6. For any integers $m, n, r \geqslant 0$, the following diagram commutes

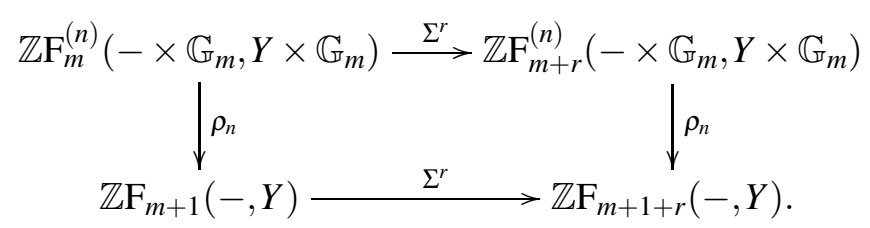

Lemma 6.7. Let $X$ and $Y$ be k-smooth schemes. Then for any integers $m$ and $n$ and any $a \in$ $\mathbb{Z F}_{m}(X, Y)$, one has $a \bigotimes\left(\mathrm{id}-e_{1}\right) \in \mathbb{Z F}_{m}^{(n)}\left(X \times \mathbb{G}_{m}, Y \times \mathbb{G}_{m}\right)$. In particular, for any integers $m$ and $n$ there is a well defined composite morphism

$$
\rho_{n} \circ\left(-\otimes\left(\mathrm{id}-e_{1}\right)\right): \mathbb{Z F}_{m}(-\times X, Y) \rightarrow \mathbb{Z F}_{m}^{(n)}\left(-\times X \times \mathbb{G}_{m}, Y \times \mathbb{G}_{m}\right) \rightarrow \mathbb{Z}_{m+1}(-\times X, Y) .
$$

Moreover, for an element $a \in \mathbb{Z F}_{m}(W \times X, Y)$ of the form $\left(Z, U,\left(\varphi_{1}, \varphi_{2}, \ldots, \varphi_{m}\right), g\right)$ one has

$$
\begin{aligned}
\rho_{n}\left(a \otimes\left(\mathrm{id}-e_{1}\right)\right) & =-\left(Z \times Z\left(t^{n+1}-t\right), U \times \mathbb{G}_{m},\left(t^{n+1}-t, \varphi_{1}, \varphi_{2}, \ldots, \varphi_{m}\right), g\right)+ \\
+ & \left(Z \times Z\left(t^{n+1}-1\right), U \times \mathbb{G}_{m},\left(t^{n+1}-1, \varphi_{1}, \varphi_{2}, \ldots, \varphi_{m}\right), g\right) \in \mathbb{Z F}_{m+1}(W \times X, Y) .
\end{aligned}
$$

Proof. Let $a \in \mathbb{Z F}_{m}(W \times X, Y)$ be the image of $\left(Z, U,\left(\varphi_{1}, \varphi_{2}, \ldots, \varphi_{m}\right), g\right) \in \operatorname{Fr}_{m}(W \times X, Y)$. Then

$$
\begin{aligned}
a \otimes\left(\mathrm{id}-e_{1}\right)=\left(Z \times \mathbb{G}_{m}, U \times \mathbb{G}_{m},\left(\varphi_{1}, \varphi_{2}, \ldots, \varphi_{m}\right),(g, t)\right)- & \\
& -\left(Z \times \mathbb{G}_{m}, U \times \mathbb{G}_{m},\left(\varphi_{1}, \varphi_{2}, \ldots, \varphi_{m}\right),\left(g, e_{1}\right)\right) \in \mathbb{Z F}_{m}\left(W \times X \times \mathbb{G}_{m}, Y \times \mathbb{G}_{m}\right),
\end{aligned}
$$

where $t$ is the coordinate function on $\mathbb{G}_{m}$. Clearly, $Z_{n}^{+}=Z \times Z\left(t^{n+1}-1\right) \subset Z \times \mathbb{G}_{m}$ and $Z_{n}^{-}=$ $Z \times Z\left(t^{n+1}-t\right) \subset Z \times \mathbb{G}_{m}$. Both sets are finite over $X$. Hence $a \bigotimes\left(\mathrm{id}-e_{1}\right) \in \mathbb{Z} \mathrm{F}_{m}^{(n)}\left(X \times \mathbb{G}_{m}, Y \times \mathbb{G}_{m}\right)$ in this case. Any element of $\mathbb{Z F}_{m}(W \times X, Y)$ is a linear combination of elements of the form $\left(Z, U,\left(\varphi_{1}, \varphi_{2}, \ldots, \varphi_{m}\right), g\right)$. This proves the first assertion of the lemma.

Computing $\rho_{n}\left(a \otimes\left(\mathrm{id}-e_{1}\right)\right)$ for $a=\left(Z, U,\left(\varphi_{1}, \varphi_{2}, \ldots, \varphi_{m}\right), g\right)$ we obtain

$$
\begin{aligned}
\rho_{n}\left(a \otimes\left(\mathrm{id}-e_{1}\right)\right) & =-\left(Z \times Z\left(t^{n+1}-t\right), U \times \mathbb{G}_{m},\left(t^{n+1}-t, \varphi_{1}, \varphi_{2}, \ldots, \varphi_{m}\right), g\right)+ \\
+ & \left(Z \times Z\left(t^{n+1}-1\right), U \times \mathbb{G}_{m},\left(t^{n+1}-1, \varphi_{1}, \varphi_{2}, \ldots, \varphi_{m}\right), g\right) \in \mathbb{Z F}_{m+1}(W \times X, Y),
\end{aligned}
$$

as was to be shown. 
Lemma 6.8. Let $X$ and $Y$ be $k$-smooth schemes. Then for every even integer $m$ and any $n$ one has

$$
\rho_{n} \circ\left(-\otimes\left(\mathrm{id}-e_{1}\right)\right) \sim(-\otimes \varepsilon): \mathbb{Z}_{m}(-\times X, Y) \rightrightarrows \mathbb{Z}_{m+1}(-\times X, Y),
$$

where $\varepsilon=\left(\{0\}, \mathbb{A}^{1},-t, c^{\prime}\right) \in \mathbb{Z F}_{1}(\mathrm{pt}, \mathrm{pt})$.

Proof. Set $\eta_{n}=\rho_{n} \circ\left(-\otimes\left(\mathrm{id}-e_{1}\right)\right)$. Take the matrix

$$
A=\left(\begin{array}{cccccc}
0 & 1 & 0 & \ldots & 0 & 0 \\
0 & 0 & 1 & \ldots & 0 & 0 \\
\vdots & \vdots & \vdots & \ddots & \vdots & \vdots \\
0 & 0 & 0 & \ldots & 0 & 1 \\
1 & 0 & 0 & \ldots & 0 & 0
\end{array}\right) \in S L_{m+1}(k)
$$

and let $A_{s} \in S L_{m+1}(k[s])$ be such that $A_{0}=i d, A_{1}=A$. Let $H_{A_{s}}$ be the $\mathbb{A}^{1}$-homotopy from Definition 4.6 between the identity and $\varphi_{A}$. By Definition 4.6 one has

$$
\eta_{n}=\rho_{n} \circ\left(-\otimes\left(\mathrm{id}-e_{1}\right)\right) \stackrel{H_{A_{s}} \circ \eta_{n}}{\longrightarrow} \varphi_{A} \circ \rho_{n} \circ\left(-\otimes\left(\mathrm{id}-e_{1}\right)\right)=\varphi_{A} \circ \eta_{n} .
$$

Set $H^{\prime}=H_{A_{s}} \circ \eta_{n}$. By Lemma 4.4 it remains to find an $H^{\prime \prime}$ such that $\varphi_{A} \circ \eta_{n} \underline{H^{\prime \prime}}(-\nabla \varepsilon)$ and set $H=H^{\prime}+H^{\prime \prime}-H_{\varphi_{A} \circ \eta_{n}}$. In this case by Lemma 4.4 one gets $\rho_{n} \circ\left(-\otimes\left(\mathrm{id}-e_{1}\right)\right)=\eta_{n} \underline{H}(-\otimes \varepsilon)$.

To construct $H^{\prime \prime}$, note that by the last statement of Lemma 6.7 one has

$$
\varphi_{A} \circ \eta_{n}=-\otimes\left[\left(Z\left(t^{n+1}-1\right), \mathbb{G}_{m}, t^{n+1}-1, c\right)-\left(Z\left(t^{n+1}-t\right), \mathbb{G}_{m}, t^{n+1}-t, c\right)\right]
$$

and $(-\otimes \varepsilon)=-\otimes\left(\{0\}, \mathbb{A}^{1},-t, c^{\prime}\right)$, where $c: \mathbb{G}_{m} \rightarrow$ pt is the canonical projection. By Lemma 4.9 one can take $H^{\prime \prime}$ to be an $\mathbb{A}^{1}$-homotopy of the form $H^{\prime \prime}=\left(-\otimes h^{\prime \prime}\right)$, where $h^{\prime \prime} \in \mathbb{Z} \mathrm{F}_{1}\left(\mathbb{A}^{1}, p t\right)$ is such that

$$
\left(Z\left(t^{n+1}-1\right), \mathbb{G}_{m}, t^{n+1}-1, c\right)-\left(Z\left(t^{n+1}-t\right), \mathbb{G}_{m}, t^{n+1}-t, c\right)=h_{0}^{\prime \prime}
$$

and

$$
h_{1}^{\prime \prime}=\left(\{0\}, \mathbb{A}^{1},-t, c^{\prime}\right) \in \mathbb{Z F}_{1}(\mathrm{pt}, \mathrm{pt}),
$$

where $c^{\prime}: \mathbb{A}^{1} \rightarrow \mathrm{pt}$ is the canonical projection. Now let us find the desired element $h^{\prime \prime}$. Since $t^{n+1}-1$ does not vanish at $t=0$, we can extend the neighborhood from $\mathbb{G}_{m}$ to $\mathbb{A}^{1}$ to get an equality,

$$
\left.\left(Z\left(t^{n+1}-1\right), \mathbb{G}_{m}, t^{n+1}-1, c\right)=\left(Z\left(t^{n+1}-1\right), \mathbb{A}^{1}, t^{n+1}-1\right), c^{\prime}\right) \in \mathbb{Z F}_{1}(p t, p t) .
$$

By Lemma 4.14 there is $h^{\prime \prime \prime} \in \mathbb{Z} \mathrm{F}_{1}\left(\mathbb{A}^{1}, p t\right)$ such that

$$
\left(Z\left(t^{n+1}-1\right), \mathbb{A}^{1}, t^{n+1}-1, c^{\prime}\right)=h_{0}^{\prime \prime \prime} \text { and } h_{1}^{\prime \prime \prime}=\left(Z\left(t^{n+1}-t\right), \mathbb{A}^{1}, t^{n+1}-t, c^{\prime}\right) \in \mathbb{Z} \mathrm{F}_{1}(p t, p t),
$$

because polynomials $t^{n+1}-t$ and $t^{n+1}-1$ have the same degree and the same leading coefficient. Using the additivity property for supports in $\mathbb{Z F}_{1}(p t, p t)$ and the second statement of Lemma 4.13, we can find an element $h^{i v} \in \mathbb{Z} \mathrm{F}_{1}\left(\mathbb{A}^{1}, p t\right)$ such that

$\left(Z\left(t^{n+1}-t\right), \mathbb{G}_{m}, t^{n+1}-t, c\right)=h_{0}^{i v}$ and $h_{1}^{i v}=\left(Z\left(t^{n+1}-t\right), \mathbb{A}^{1}, t^{n+1}-t, c^{\prime}\right)-\left(\{0\}, \mathbb{A}^{1},-t, c^{\prime}\right) \in \mathbb{Z} \mathrm{F}_{1}(p t, p t)$

Set $h^{\prime \prime}:=h^{\prime \prime \prime}-h^{i v} \in \mathbb{Z} \mathrm{F}_{1}\left(\mathbb{A}^{1}, p t\right)$. Then $h^{\prime \prime}$ is the desired element.

Set $H^{\prime \prime}=\left(-\bigotimes h^{\prime \prime}\right)$ and $H=H^{\prime}+H^{\prime \prime}-H_{\varphi_{A} \circ \eta_{n}}$. Then $H$ is the desired $\mathbb{A}^{1}$-homotopy. That is

$$
\rho_{n} \circ\left(-\nabla\left(\mathrm{id}-e_{1}\right)\right) \frac{H}{-}(-\nabla \varepsilon)
$$

and our statement follows. 


\section{THEOREM C}

The main purpose of this section is to prove Theorem C. We sometimes identify simplicial abelian groups with chain complexes concentrated in non-negative degrees by using the Dold-Kan correspondence.

Lemma 7.1. Let $X$ and $Y$ be $k$-smooth schemes and $m, r, N \geqslant 0$ be integers. Then for any Moore cycle $a \in \mathbb{Z F}_{m}\left(\Delta^{r} \times X, Y\right)$ of the simplicial abelian group $\mathbb{Z F}_{m}\left(\Delta^{\bullet} \times X, Y\right)$, one has a $\otimes\left(i d-e_{1}\right) \in$ $\mathbb{Z F}_{m}^{(N)}\left(\Delta^{r} \times X \times \mathbb{G}_{m}, Y \times \mathbb{G}_{m}\right)$. Moreover, $\rho_{N}\left(a \otimes\left(i d-e_{1}\right)\right)$ is a Moore cycle. The homology classes of Moore cycles

$$
a \otimes \varepsilon \text { and } \rho_{N}\left(a \otimes\left(i d-e_{1}\right)\right)
$$

coincide in $\mathbb{Z F}_{m+1}\left(\Delta^{\bullet} \times X, Y\right)$.

Proof. The element $a \otimes\left(i d-e_{1}\right)$ is in $\mathbb{Z F}_{m}^{(N)}\left(\Delta^{r} \times X \times \mathbb{G}_{m}, Y \times \mathbb{G}_{m}\right)$ by Lemma 6.7. Since $\left.\mathbb{Z F}_{m}^{(N)}\left(-\times \mathbb{G}_{m}, Y \times \mathbb{G}_{m}\right)\right)$ is a presheaf, then $\partial_{i}\left(a \otimes\left(i d-e_{1}\right)\right) \in \mathbb{Z F}_{m}^{(N)}\left(\Delta^{r} \times X \times \mathbb{G}_{m}, Y \times \mathbb{G}_{m}\right)$. Since the morphism $\rho_{N}$ is a morphism of presheaves, then

$$
\partial_{i}\left(\rho_{N}\left(a \otimes\left(i d-e_{1}\right)\right)\right)=\rho_{N}\left(\partial_{i}\left(a \otimes\left(i d-e_{1}\right)\right)=\rho_{N}\left(\partial_{i}(a) \otimes\left(i d-e_{1}\right)\right)=0 .\right.
$$

This proves the first assertion of the lemma.

By Lemma 6.8 the morphism

$$
a^{\prime} \mapsto \rho_{N}\left(a^{\prime} \otimes\left(\operatorname{id}_{\mathbb{G}_{m}}-e_{1}\right)\right): \mathbb{Z}_{m}(-\times X, Y) \rightarrow \mathbb{Z F}_{m}^{(N)}(-\times X, Y) \rightarrow \mathbb{Z F}_{m+1}(-\times X, Y)
$$

is $\mathbb{A}^{1}$-homotopic to the morphism $a^{\prime} \mapsto a^{\prime} \otimes \varepsilon$. Thus the corresponding morphisms of the simplicial abelian groups $\mathbb{Z F}_{m}\left(\Delta^{\bullet} \times X, Y\right) \rightrightarrows \mathbb{Z F}_{m+1}\left(\Delta^{\bullet} \times X, Y\right)$ induce the same morphisms on homology. Hence the homology class of the Moore cycle $\rho_{N}\left(a \otimes\left(\mathrm{id}_{\mathbb{G}_{m}}-e_{1}\right)\right)$ coincides with the homology class of the Moore cycle $a \bigotimes \varepsilon$.

Lemma 7.2. One has $\varepsilon \otimes \varepsilon \sim \Sigma^{2}$ in $\mathbb{Z F}_{2}$ (pt, pt). Moreover, for any integer $r \geqslant 0$ one has $\varepsilon \otimes \varepsilon \otimes \Sigma^{r} \sim$ $\Sigma^{2+r}$ in $\mathbb{Z F}_{2+r}(\mathrm{pt}, \mathrm{pt})$.

Proof. Let $c: \mathbb{A}^{1} \times \mathbb{A}^{2} \rightarrow \mathrm{pt}$ be the structure morphism. Take the matrix

$$
A=\left(\begin{array}{cc}
0 & -1 \\
-1 & 0
\end{array}\right) \in S L_{2}(k) .
$$

There is an $A_{s} \in S L_{2}(k[s])$ such that $A_{0}=i d, A_{1}=A$. Take

$$
h_{s}=\left(\mathbb{A}^{1} \times 0, \mathbb{A}^{1} \times \mathbb{A}^{2}, A_{s} \circ\left(t_{1}, t_{2}\right), c\right) \in \mathbb{Z F}_{2}\left(\mathbb{A}^{1}, \mathrm{pt}\right) .
$$

Clearly, $h_{0}=\Sigma^{2}$ and $h_{1}=\varepsilon \otimes \varepsilon$. The first assertion is proved. To prove the second one take the element $h_{s} \otimes \Sigma^{r} \in \mathbb{Z F}_{2+r}\left(\mathbb{A}^{1}, \mathrm{pt}\right)$. Then $h_{0} \otimes \Sigma^{r}=\Sigma^{2+r}$ and $h_{1} \otimes \Sigma^{r}=\varepsilon \otimes \varepsilon \otimes \Sigma^{r}$.

Corollary 7.3. Let $X$ and $Y$ be $k$-smooth schemes and $m \geqslant 0$ be an integer. Then,

$$
\left(-\bigotimes \varepsilon^{2}\right) \sim\left(-\otimes \Sigma^{2}\right): \mathbb{Z F}_{m}(-\times X, Y) \rightrightarrows \mathbb{Z F}_{m+2}(-\times X, Y)
$$

and

$$
\left(-\otimes \varepsilon^{2} \otimes \Sigma^{r}\right) \sim\left(-\otimes \Sigma^{2+r}\right): \mathbb{Z}_{m}(-\times X, Y) \rightrightarrows \mathbb{Z}_{m+2+r}(-\times X, Y) .
$$

Therefore the first pair of maps produces the same maps on homology

$$
H_{*}\left(\mathbb{Z F}_{m}\left(\Delta^{\bullet} \times X, Y\right)\right) \rightrightarrows H_{*}\left(\mathbb{Z F}_{m+2}\left(\Delta^{\bullet} \times X, Y\right)\right) .
$$

Similarly, the second pair of maps gives the same maps on homology

$$
H_{*}\left(\mathbb{Z F}_{m}\left(\Delta^{\bullet} \times X, Y\right)\right) \rightrightarrows H_{*}\left(\mathbb{Z F}_{m+2+r}\left(\Delta^{\bullet} \times X, Y\right)\right) .
$$


Lemma 7.4. Let $X$ and $Y$ be $k$-smooth schemes and $m \geqslant 0$ be an integer. Then for any integer $r \geqslant 0$ one has

$$
\begin{aligned}
\operatorname{Ker}\left[-\otimes\left(\operatorname{id}_{\mathbb{G}_{m}}-e_{1}\right): H_{r}\left(\mathbb{Z}_{m}\left(\Delta^{\bullet} \times X, Y\right)\right) \rightarrow H_{r}\left(\mathbb{Z F}_{m}\left(\left(\Delta^{\bullet} \times X\right) \wedge\left(\mathbb{G}_{m}, 1\right), Y \wedge\left(\mathbb{G}_{m}, 1\right)\right)\right)\right] \subseteq \\
\subseteq \operatorname{Ker}\left[\left(-\otimes \Sigma^{2}\right): H_{r}\left(\mathbb{Z F}_{m}\left(\Delta^{\bullet} \times X, Y\right)\right) \rightarrow H_{r}\left(\mathbb{Z F}_{m+2}\left(\Delta^{\bullet} \times X, Y\right)\right)\right] .
\end{aligned}
$$

Proof. Consider the associated Moore complexes. Assume that

$$
a \in \mathbb{Z} \mathrm{F}_{m}\left(\Delta^{r} \times X, Y\right)
$$

is a Moore cycle for which $a \otimes\left(\operatorname{id}_{\mathbb{G}_{m}}-e_{1}\right)$ is a boundary, i.e., there exists $b \in \mathbb{Z} \mathrm{F}_{m}\left(\left(\Delta^{r+1} \times X\right) \times\right.$ $\left.\left.\mathbb{G}_{m}, Y \times \mathbb{G}_{m}\right)\right)$ such that $\partial_{i}(b)=0$ for $i=0,1, \ldots, r$ and $\partial_{r+1}(b)=a \bigotimes\left(\operatorname{id}_{\mathbb{G}_{m}}-e_{1}\right)$. By Lemma 6.4 there exists an $N$ such that $b \in \mathbb{Z F}_{m}^{(N)}\left(\Delta^{r+1} \times X \times \mathbb{G}_{m}, Y \times \mathbb{G}_{m}\right)$. Since $\mathbb{Z} \mathrm{F}_{m}^{(N)}\left(-\times \mathbb{G}_{m}, Y \times \mathbb{G}_{m}\right)$ is a presheaf, then $\partial_{i}(b) \in \mathbb{Z} \mathrm{F}_{m}^{(N)}\left(\Delta^{r} \times X \times \mathbb{G}_{m}, Y \times \mathbb{G}_{m}\right)$. Since $\rho_{N}$ is a presheaf morphism $\mathbb{Z} \mathrm{F}_{m}^{(N)}(-\times$ $\left.X \times \mathbb{G}_{m}, Y \times \mathbb{G}_{m}\right) \rightarrow \mathbb{Z F}_{m+1}(-\times X, Y)$, one has $\partial_{i}\left(\rho_{N}(b)\right)=\rho_{N}\left(\partial_{i}(b)\right)$. Thus,

$$
\partial_{i}\left(\rho_{N}(b)\right)=\rho_{N}\left(\partial_{i}(b)\right)=0 \text { for } 0 \leqslant i \leqslant r,
$$

$$
\partial_{r+1}\left(\rho_{N}(b)\right)=\rho_{N}\left(\partial_{n+1}(b)\right)=\rho_{N}\left(a \otimes\left(\mathrm{id}_{\mathbb{G}_{m}}-e_{1}\right)\right) .
$$

We see that the homology class of the Moore cycle $\rho_{N}\left(a \otimes\left(\operatorname{id}_{\mathbb{G}_{m}}-e_{1}\right)\right)$ vanishes. By Lemma 7.1

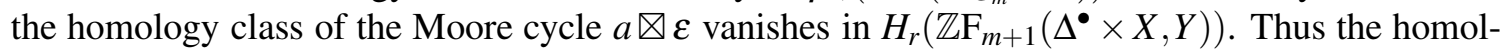
ogy class of the Moore cycle $a \otimes \varepsilon \otimes \varepsilon$ vanishes in $H_{r}\left(\mathbb{Z}_{m+2}\left(\Delta^{\bullet} \times X, Y\right)\right)$. By Corollary 7.3 the homology class of $a \otimes \Sigma^{2}$ vanishes in $H_{r}\left(\mathbb{Z F}_{m+2}\left(\Delta^{\bullet} \times X, Y\right)\right)$, too.

Lemma 7.5. Let $X$ and $Y$ be $k$-smooth schemes and $m, r \geqslant 0$ be integers. Let $n$ be the integer from Lemma 5.4. Then for any Moore cycle $a \in \mathbb{Z F}_{m}\left(\left(\Delta^{r} \times X\right) \wedge\left(\mathbb{G}_{m}, 1\right), Y \wedge\left(\mathbb{G}_{m}, 1\right)\right)$ there exists an integer $N$ such that the element $\rho_{N}(a)$ is defined and the homology class of the Moore cycle

$$
\Sigma^{2 n}\left(\rho_{N}(a)\right) \otimes\left(i d-e_{1}\right) \in \mathbb{Z F}_{m+2 n+1}\left(\left(\Delta^{r} \times X\right) \wedge\left(\mathbb{G}_{m}, 1\right), Y \wedge\left(\mathbb{G}_{m}, 1\right)\right)
$$

coincides with the homology class of the Moore cycle $\Sigma^{2 n}(a \otimes \varepsilon)$.

Proof. Set $a^{\prime}=a \otimes\left(i d-e_{1}\right)$. Let $H$ be the $\mathbb{A}^{1}$-homotopy from Lemma 5.4. Consider the element

$$
H\left(a^{\prime}\right) \in \mathbb{Z F}_{m+2 n}\left(\left(\Delta^{r} \times X\right) \times \mathbb{G}_{m} \times \mathbb{G}_{m}, Y \times \mathbb{G}_{m} \times \mathbb{G}_{m}\right) .
$$

By Lemma 6.4 there is an integer $N$ such that

$$
a \in \mathbb{Z} \mathrm{F}_{m}^{(N)}\left(\left(\Delta^{r} \times X\right) \times \mathbb{G}_{m}, Y \times \mathbb{G}_{m}\right)
$$

and

$$
H\left(a^{\prime}\right) \in \mathbb{Z F}_{m+2 n}^{(N)}\left(\left(\Delta^{r} \times X\right) \times \mathbb{G}_{m} \times \mathbb{G}_{m} \times \mathbb{A}^{1}, Y \times \mathbb{G}_{m} \times \mathbb{G}_{m}\right) .
$$

Since $a^{\prime}$ is a Moore cycle and $H$ is a presheaf morphism, the element $H\left(a^{\prime}\right)$ is a Moore cycle in $\mathbb{Z F}_{m+2 n}\left(\left(\Delta^{\bullet} \times X\right) \times \mathbb{G}_{m} \times \mathbb{G}_{m} \times \mathbb{A}^{1}, Y \times \mathbb{G}_{m} \times \mathbb{G}_{m}\right)$. Since

$$
\mathbb{Z} \mathrm{F}_{m+2 n}^{(N)}\left((-\times X) \times \mathbb{G}_{m} \times \mathbb{G}_{m} \times \mathbb{A}^{1}, Y \times \mathbb{G}_{m} \times \mathbb{G}_{m}\right)
$$

is a subpresheaf of $\mathbb{Z F}_{m}\left((-\times X) \times \mathbb{G}_{m} \times \mathbb{G}_{m} \times \mathbb{A}^{1}, Y \times \mathbb{G}_{m} \times \mathbb{G}_{m}\right)$, it follows that $H\left(a^{\prime}\right)$ is a Moore cycle in $\mathbb{Z F}_{m+2 n}^{(N)}\left(\left(\Delta^{\bullet} \times X\right) \times \mathbb{G}_{m} \times \mathbb{G}_{m} \times \mathbb{A}^{1}, Y \times \mathbb{G}_{m} \times \mathbb{G}_{m}\right)$.

Applying the presheaf morphism

$\rho_{N}: \mathbb{Z F}_{m+2 n}^{(N)}\left((-\times X) \times \mathbb{G}_{m} \times \mathbb{G}_{m} \times \mathbb{A}^{1}, Y \times \mathbb{G}_{m} \times \mathbb{G}_{m}\right) \rightarrow \mathbb{Z F}_{m+2 n+1}\left((-\times X) \times \mathbb{G}_{m} \times \mathbb{A}^{1}, Y \times \mathbb{G}_{m}\right)$ to the Moore cycle $H\left(a^{\prime}\right)$, we get a Moore cycle

$$
\rho_{N}\left(H\left(a^{\prime}\right)\right) \in \mathbb{Z F}_{m+2 n+1}\left(\left(\Delta^{r} \times X\right) \times \mathbb{G}_{m} \times \mathbb{A}^{1}, Y \times \mathbb{G}_{m}\right) .
$$


Hence $i_{0}^{*}\left(\rho_{N}\left(H\left(a^{\prime}\right)\right)\right) \in \mathbb{Z F}_{m+2 n+1}\left(\left(\Delta^{r} \times X\right) \times \mathbb{G}_{m}, Y \times \mathbb{G}_{m}\right)$ and $i_{1}^{*}\left(\rho_{N}\left(H\left(a^{\prime}\right)\right)\right) \in \mathbb{Z} \mathrm{F}_{m+2 n+1}\left(\left(\Delta^{r} \times\right.\right.$ $\left.X) \times \mathbb{G}_{m}, Y \times \mathbb{G}_{m}\right)$ are Moore cycles, too. Furthermore,

$$
i_{0}^{*}\left(\rho_{N}\left(H\left(a^{\prime}\right)\right)\right)=\rho_{N}\left(i_{0}^{*}\left(H\left(a^{\prime}\right)\right)\right)=\rho_{N}\left(\Sigma^{2 n}\left(a^{\prime}\right)\right)=\Sigma^{2 n}\left(\rho_{N}\left(a^{\prime}\right)\right)
$$

and

$$
i_{1}^{*}\left(\rho_{N}\left(H\left(a^{\prime}\right)\right)\right)=\rho_{N}\left(i_{1}^{*}\left(H\left(a^{\prime}\right)\right)\right)=\rho_{N}\left(\Sigma^{2 n}\left[\left(\operatorname{id}_{Y} \times \tau\right) \circ a^{\prime} \circ\left(\operatorname{id}_{X} \times \tau\right)\right]\right) .
$$

The two morphisms

$$
\left.\left.i_{0}^{*}, i_{1}^{*}: \mathbb{Z F}_{n+2 m+1}\left(\left(\Delta^{\bullet} \times X\right) \times \mathbb{G}_{m} \times \mathbb{A}^{1}, Y \times \mathbb{G}_{m}\right)\right) \rightrightarrows \mathbb{Z F}_{n+2 m+1}\left(\left(\Delta^{\bullet} \times X\right) \times \mathbb{G}_{m}, Y \times \mathbb{G}_{m}\right)\right)
$$

of simplicial abelian groups induce the same morphisms on homology. The element $\rho_{N}\left(\left(H\left(a^{\prime}\right)\right)\right.$ is a Moore cycle. Thus the homological classes of the Moore cycles $i_{0}^{*}\left(\rho_{N}\left(H\left(a^{\prime}\right)\right)\right)$ and $i_{1}^{*}\left(\rho_{N}\left(H\left(a^{\prime}\right)\right)\right)$ coincide in $\left.H_{r}\left(\mathbb{Z F}_{n+2 m+1}\left(\left(\Delta^{\bullet} \times X\right) \times \mathbb{G}_{m}, Y \times \mathbb{G}_{m}\right)\right)\right)$.

By Lemma 6.6 one has $\rho_{N}\left(\Sigma^{2 n}\left(a^{\prime}\right)\right)=\Sigma^{2 n}\left(\rho_{N}\left(a^{\prime}\right)\right)$. Thus the first homological class is the class of $\Sigma^{2 n}\left(\rho_{N}\left(a^{\prime}\right)\right)=\Sigma^{2 n}\left(\rho_{N}\left(a \otimes\left(i d-e_{1}\right)\right)\right)$. By Lemma 7.1 the latter homological class coincides with the class of the element $\Sigma^{2 n}(a \otimes \varepsilon)$.

The element $i_{1}^{*}\left(\rho_{N}\left(H\left(a^{\prime}\right)\right)\right)$ coincides with $\rho_{N}\left(\Sigma^{2 n}\left[\left(\operatorname{id}_{Y} \times \tau\right) \circ\left(a \otimes\left(i d-e_{1}\right)\right) \circ\left(\operatorname{id}_{X} \times \tau\right)\right]\right)$. By Lemma 6.6 the latter element coincides with

$$
\Sigma^{2 n}\left(\rho_{N}\left[\left(\operatorname{id}_{Y} \times \tau\right) \circ\left(a \otimes\left(i d-e_{1}\right)\right) \circ\left(\operatorname{id}_{X} \times \tau\right)\right]\right)=\Sigma^{2 n}\left[\rho_{N}(a) \otimes\left(i d-e_{1}\right)\right] .
$$

Hence the homological classes $\Sigma^{2 n}(a \otimes \varepsilon)$ and $\left[\Sigma^{2 n}\left[\rho_{N}(a) \otimes\left(i d-e_{1}\right)\right]\right]$ coincide in $H_{r}\left(\mathbb{Z F}_{n+2 m+1}\left(\left(\Delta^{\bullet} \times X\right) \times \mathbb{G}_{m}, Y \times \mathbb{G}_{m}\right)\right)$. Finally, the complex $\mathbb{Z} \mathrm{F}_{n+2 m+1}\left(\left(\Delta^{\bullet} \times X\right) \wedge\left(\mathbb{G}_{m}, 1\right), Y \wedge\right.$

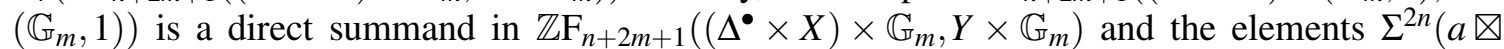
$\varepsilon), \Sigma^{2 n}\left(\rho_{N}(a) \otimes\left(i d-e_{1}\right)\right)$ are in $\mathbb{Z} F_{n+2 m+1}\left(\left(\Delta^{r} \times X\right) \wedge\left(\mathbb{G}_{m}, 1\right), Y \wedge\left(\mathbb{G}_{m}, 1\right)\right)$. Hence the homological classes $\left[\Sigma^{2 n}\left[\rho_{N}(a) \otimes\left(i d-e_{1}\right)\right]\right]$ and $\left[\Sigma^{2 n}(a \otimes \varepsilon)\right]$ coincide in $H_{r}\left(\mathbb{Z F}_{n+2 m+1}\left(\left(\Delta^{\bullet} \times X\right) \wedge\left(\mathbb{G}_{m}, 1\right), Y \wedge\right.\right.$ $\left.\left(\mathbb{G}_{m}, 1\right)\right)$.

Lemma 7.6. Let $X$ and $Y$ be $k$-smooth schemes and $m, r \geqslant 0$ be integers. Let $n$ be the integer from Lemma 5.4. Then

$$
\begin{aligned}
\operatorname{Im}\left[\left(-\otimes \Sigma^{2 n+2}\right): H_{r}\right. & \left(\mathbb{Z F}_{m}\left(\left(\Delta^{\bullet} \times X\right) \wedge\left(\mathbb{G}_{m}, 1\right), Y \wedge\left(\mathbb{G}_{m}, 1\right)\right)\right) \rightarrow \\
& \left.\rightarrow H_{r}\left(\mathbb{Z F}_{m+2 n+2}\left(\left(\Delta^{\bullet} \times X\right) \wedge\left(\mathbb{G}_{m}, 1\right), Y \wedge\left(\mathbb{G}_{m}, 1\right)\right)\right)\right] \subseteq \\
\operatorname{Im}\left[\left(-\otimes\left(\operatorname{id}_{\mathbb{G}_{m}}-e_{1}\right)\right):\right. & \left.H_{r}\left(\mathbb{Z}_{m+2 n+2}\left(\Delta^{\bullet} \times X, Y\right)\right) \rightarrow H_{r}\left(\mathbb{Z}_{m+2 n+2}\left(\left(\Delta^{\bullet} \times X\right) \wedge\left(\mathbb{G}_{m}, 1\right), Y \wedge\left(\mathbb{G}_{m}, 1\right)\right)\right)\right] .
\end{aligned}
$$

Proof. Take a Moore cycle $a^{\prime} \in \mathbb{Z F}_{m}\left(\left(\Delta^{r} \times X\right) \wedge\left(\mathbb{G}_{m}, 1\right), Y \wedge\left(\mathbb{G}_{m}, 1\right)\right)$. Then the element $a:=a^{\prime} \otimes \varepsilon$ is a Moore cycle in $\mathbb{Z F}_{m+1}\left(\left(\Delta^{r} \times X\right) \wedge\left(\mathbb{G}_{m}, 1\right), Y \wedge\left(\mathbb{G}_{m}, 1\right)\right)$. By Lemma 7.5 the homology classes of $\Sigma^{2 n}(a \otimes \varepsilon)$ and $\Sigma^{2 n}\left(\rho_{N}(a)\right) \otimes\left(i d-e_{1}\right)$ coincide in

$$
H_{r}\left(\mathbb{Z F}_{m+2+2 n}\left(\left(\Delta^{\bullet} \times X\right) \wedge\left(\mathbb{G}_{m}, 1\right), Y \wedge\left(\mathbb{G}_{m}, 1\right)\right)\right) .
$$

By Corollary 7.3 the homology classes of $\Sigma^{2 n}(a \otimes \varepsilon)=\Sigma^{2 n}\left(a^{\prime} \otimes \varepsilon \otimes \varepsilon\right)$ and $\Sigma^{2 n+2}\left(a^{\prime}\right)$ coincide. Hence the homology classes of $\Sigma^{2 n+2}\left(a^{\prime}\right)$ and $\Sigma^{2 n}\left(\rho_{N}\left(a^{\prime} \otimes \varepsilon\right)\right) \otimes\left(i d-e_{1}\right)$ coincide in $H_{r}\left(\mathbb{Z F}_{m+2+2 n}\left(\left(\Delta^{\bullet} \times X\right) \wedge\left(\mathbb{G}_{m}, 1\right), Y \wedge\left(\mathbb{G}_{m}, 1\right)\right)\right)$.

We are now in a position to prove Theorem C.

Theorem C. Let $X$ and $Y$ be k-smooth schemes. Then

$$
-\otimes\left(\operatorname{id}_{\mathbb{G}_{m}}-e_{1}\right): \mathbb{Z} \mathrm{F}\left(\Delta^{\bullet} \times X, Y\right) \rightarrow \mathbb{Z} \mathrm{F}\left(\left(\Delta^{\bullet} \times X\right) \wedge\left(\mathbb{G}_{m}, 1\right), Y \wedge\left(\mathbb{G}_{m}, 1\right)\right)
$$

is a quasi-isomorphism of complexes of abelian groups. 
Proof. The theorem follows from Lemmas 7.4 and 7.6.

In more detail, first prove that the morphism $-\otimes\left(\operatorname{id}_{\mathbb{G}_{m}}-e_{1}\right)$ induces an epimophism on homology groups. For this take an integer $r \geqslant 0$ and an element $a \in H_{r}\left(\mathbb{Z F}\left(\left(\Delta^{\bullet} \times X\right) \wedge\left(\mathbb{G}_{m}, 1\right), Y \wedge\right.\right.$ $\left.\left.\left(\mathbb{G}_{m}, 1\right)\right)\right)$. We will find an element $b \in H_{r}\left(\mathbb{Z} \mathrm{F}\left(\Delta^{\bullet} \times X, Y\right)\right)$ such that $b \otimes\left(\operatorname{id}_{\mathbb{G}_{m}}-e_{1}\right)=a$. Note that there exist an integer $m \geqslant 0$ and an element $a_{m} \in H_{r}\left(\mathbb{Z F}_{m}\left(\left(\Delta^{\bullet} \times X\right) \wedge\left(\mathbb{G}_{m}, 1\right), Y \wedge\left(\mathbb{G}_{m}, 1\right)\right)\right)$ which is a lift of the element $a$. Let $n$ be the integer from Lemma 5.4. By Lemma 7.6 there exists an element $b_{m+2 n+2} \in H_{r}\left(\mathbb{Z F}_{m+2 n+2}\left(\Delta^{\bullet} \times X, Y\right)\right)$ such that

$$
b_{m+2 n+2} \otimes\left(\operatorname{id}_{\mathbb{G}_{m}}-e_{1}\right)=a_{m} \otimes \Sigma^{2 n+2} \in H_{r}\left(\mathbb{Z}_{m+2 n+2}\left(\left(\Delta^{\bullet} \times X\right) \wedge\left(\mathbb{G}_{m}, 1\right), Y \wedge\left(\mathbb{G}_{m}, 1\right)\right)\right) .
$$

Let $b$ be the image of $b_{m+2 n+2}$ in $H_{r}\left(\mathbb{Z} \mathrm{F}\left(\Delta^{\bullet} \times X, Y\right)\right)$. Clearly, $b \otimes\left(\operatorname{id}_{\mathbb{G}_{m}}-e_{1}\right)=a$ in $H_{r}\left(\mathbb{Z} \mathrm{F}\left(\left(\Delta^{\bullet} \times\right.\right.\right.$ $\left.\left.X) \wedge\left(\mathbb{G}_{m}, 1\right), Y \wedge\left(\mathbb{G}_{m}, 1\right)\right)\right)$. Thus the morphism $-\mathbb{\nabla}\left(\mathrm{id}_{\mathbb{G}_{m}}-e_{1}\right)$ induces an epimophism on homology. The fact that the morphism $-\otimes\left(\operatorname{id}_{\mathbb{G}_{m}}-e_{1}\right)$ induces a monomophism on homology is proved in a similar fashion. Theorem $\mathrm{C}$ is proved.

\section{APPENDIX A.}

The main goal of this section is to prove Theorem D.

Let $(\mathscr{V}, \otimes)$ be a closed symmetric monoidal category and $\mathscr{C}$ is a bicomplete category which is tensored and cotensored over $\mathscr{V}$. Then for every $V \in \mathscr{V}$ and $C \in \mathscr{C}$ there are defined objects $V \otimes C, C \otimes V, \underline{\operatorname{Hom}}(V, C)$ of $\mathscr{C}$. They are all functorial in $V$ and $C$. Moreover, for every morphism $r: V \rightarrow V^{\prime}$ in $\mathscr{V}$ the square in $\mathscr{C}$

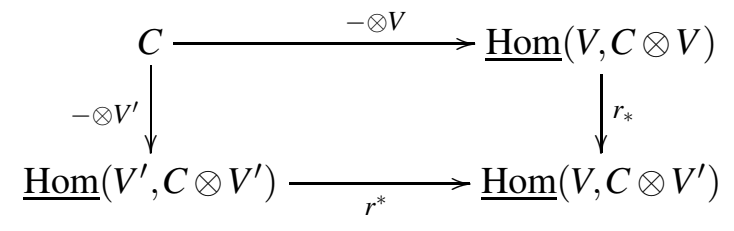

is commutative.

As an important example, $\mathscr{V}$ is the category of simplicial objects $s \operatorname{Pre}\left(\mathbb{Z F}_{0}(k)\right)$ in the category $\operatorname{Pre}\left(\mathbb{Z F}_{0}(k)\right)$ and $\mathscr{C}$ is the category $\operatorname{Pre}_{A b}\left(\mathbb{Z F}_{*}(k)\right)$ of simplicial objects in $\operatorname{Pre}_{A b}\left(\mathbb{Z} \mathrm{F}_{*}(k)\right)$. The General Framework of p. 6 is then immediately extended to this couple $(\mathscr{V}, \mathscr{C})$. Recall that the functor $\mathbb{Z} \mathrm{F}_{*}(k) \times \mathbb{Z} \mathrm{F}_{0}(k) \stackrel{\bigotimes}{\rightarrow} \mathbb{Z} \mathrm{F}_{*}(k)$ takes $(X, Y)$ to $X \times Y$. As usual, the Yoneda embedding identifies the category simplicial objects in $\mathbb{Z F}_{0}(k)$ with a full subcategory of $\operatorname{sPre}_{A b}\left(\mathbb{Z F}_{0}(k)\right)$.

The following lemma is obvious.

Lemma A.1. Suppose in the diagram (7) the morphisms $r_{*}, r^{*}$ and $-\otimes V^{\prime}$ are sectionwise weak equivalences, then the morphism $-\otimes V$ is a sectionwise weak equivalence.

As it is shown in [GP1, Section 5], the category of framed correspondences of level zero $\operatorname{Fr}_{0}(k)$ has an action by finite pointed sets $S \otimes K:=\bigsqcup_{K \backslash *} S$ with $S \in S m / k$ and $K$ a finite pointed set. The cone of $S$ is the simplicial object $S \otimes I$ in $\operatorname{Fr}_{0}(k)$, where $(I, 1)$ is the pointed simplicial set $\Delta[1]$ with basepoint 1 . There is a natural morphism $i_{0}: S \rightarrow S \otimes I$ in $\Delta^{\mathrm{op}} \operatorname{Fr}_{0}(k)$. Let pt $\stackrel{e_{1}}{\rightarrow} \mathbb{G}_{m}$ be the point 1 in $\mathbb{G}_{m}(k)$. Then $\mathbb{G}_{m}^{\wedge 1}$ is the simplicial object in $\operatorname{Fr}_{0}(k)$ which is obtained by taking the pushout of the diagram $\mathbb{G}_{m} \stackrel{e_{1}}{\longleftarrow} \mathrm{pt} \stackrel{i_{0}}{\hookrightarrow} \mathrm{pt} \otimes I$ in $\Delta^{\mathrm{op}} \operatorname{Fr}_{0}(k)$.

Let $L: \mathrm{Fr}_{0} \rightarrow \mathbb{Z F}_{0}$ be the canonical functor which is the identity on objects and which takes a morphism $\varphi \in \operatorname{Fr}_{0}(Y, X)$ to the class $1 \cdot \varphi \in \mathbb{Z F}_{0}(Y, X)$. If we apply the functor $L$ to $\mathbb{G}_{m}^{\wedge 1}$, we get an object in $\operatorname{sPre}_{A b}\left(\mathbb{Z F}_{0}(k)\right)$ denoted by $\mathbb{Z} \mathrm{F}_{0}\left(\mathbb{G}_{m}^{\wedge 1}\right)$. 
Put $\mathbb{Z F}_{0}\left(\mathbb{G}_{m}, 1\right)=\mathbb{Z F}_{0}\left(\mathbb{G}_{m}\right) / \operatorname{Im}\left(e_{1, *}\right)=\operatorname{Ker}\left(e_{1}^{*}\right)$. There is a unique morphism $r: \mathbb{Z F}_{0}\left(\mathbb{G}_{m}^{\wedge 1}\right) \rightarrow$ $\mathbb{Z} \mathrm{F}_{0}\left(\mathbb{G}_{m}, 1\right)$ which restricts to the quotient map $q: \mathbb{Z F}_{0}\left(\mathbb{G}_{m}\right) \rightarrow \mathbb{Z F}_{0}\left(\mathbb{G}_{m}\right) / \operatorname{Im}\left(e_{1, *}\right)$ on $\mathbb{Z} \mathrm{F}_{0}\left(\mathbb{G}_{m}\right)$ and which restricts to the zero map on $\mathrm{pt} \otimes I$.

The following lemma is straightforward and left to the reader.

Lemma A.2. $\mathbb{Z F}(X) \otimes \mathbb{Z F}_{0}\left(\mathbb{G}_{m}, 1\right)=\mathbb{Z F}\left(X \wedge\left(\mathbb{G}_{m}, 1\right)\right), \mathbb{Z} \mathrm{F}(X) \otimes \mathbb{Z F}_{0}\left(\mathbb{G}_{m}^{\wedge 1}\right)=\mathbb{Z F}\left(X \times \mathbb{G}_{m}^{\wedge 1}\right)$.

Lemma A.3. The morphisms

$$
\begin{aligned}
& \left.\quad r_{*}: \underline{\operatorname{Hom}}\left(\mathbb{Z F}_{0}\left(\mathbb{G}_{m}^{\wedge 1}\right), C_{*}\left(\mathbb{Z F}(X) \otimes \mathbb{Z F}_{0}\left(\mathbb{G}_{m}^{\wedge 1}\right)\right)\right) \rightarrow \underline{\operatorname{Hom}}\left(\mathbb{Z F}_{0}\left(\mathbb{G}_{m}^{\wedge 1}\right), C_{*}\left(\mathbb{Z F}(X) \otimes \mathbb{Z F}_{0}\left(\mathbb{G}_{m}, 1\right)\right)\right)\right) \\
& \left.r^{*}: \underline{\operatorname{Hom}}\left(\mathbb{Z} \mathrm{F}_{0}\left(\mathbb{G}_{m}, 1\right), C_{*}\left(\mathbb{Z F}(X) \otimes \mathbb{Z F}_{0}\left(\mathbb{G}_{m}, 1\right)\right)\right) \rightarrow \underline{\operatorname{Hom}}\left(\mathbb{Z F}_{0}\left(\mathbb{G}_{m}^{\wedge 1}\right), C_{*}\left(\mathbb{Z F}(X) \otimes \mathbb{Z F}_{0}\left(\mathbb{G}_{m}, 1\right)\right)\right)\right) \\
& \text { are sectionwise weak equivalences. }
\end{aligned}
$$

Proof. It is easy to see that the morphisms

$$
\begin{gathered}
r: \mathbb{Z F}_{0}\left(\mathbb{G}_{m}^{\wedge 1}\right) \rightarrow \mathbb{Z F}_{0}\left(\mathbb{G}_{m}, 1\right), \\
i d \otimes r: \mathbb{Z} \mathrm{F}(X) \otimes \mathbb{Z F}_{0}\left(\mathbb{G}_{m}^{\wedge 1}\right) \rightarrow \mathbb{Z} \mathrm{F}(X) \otimes \mathbb{Z} \mathrm{F}_{0}\left(\mathbb{G}_{m}, 1\right), \\
i d \otimes r: C_{*}\left(\mathbb{Z} \mathrm{F}(X) \otimes \mathbb{Z} \mathrm{F}_{0}\left(\mathbb{G}_{m}^{\wedge 1}\right)\right) \rightarrow C_{*}\left(\mathbb{Z} \mathrm{F}(X) \otimes \mathbb{Z F}_{0}\left(\mathbb{G}_{m}, 1\right)\right)
\end{gathered}
$$

are sectionwise weak equivalences. The lemma now follows.

Theorem C, Lemma A.1 and Lemma A.3 imply the following

Corollary A.4. The morphism

$$
-\otimes \mathbb{G}_{m}^{\wedge 1}: C_{*} \mathbb{Z} \mathrm{F}(X) \rightarrow \underline{\operatorname{Hom}}\left(\mathbb{G}_{m}^{\wedge 1}, C_{*} \mathbb{Z} \mathrm{F}\left(X \times \mathbb{G}_{m}^{\wedge 1}\right)\right)
$$

is a sectionwise weak equivalence in $\operatorname{sPr}_{A b}\left(\mathbb{Z F}_{*}(k)\right)$.

We are now in a position to prove the following

Theorem D. The morphism $c_{0}: L M_{f r}(X) \rightarrow \underline{\operatorname{Hom}}\left(\mathbb{G}, L M_{f r}\left(X \times \mathbb{G}_{m}^{\wedge 1}\right)\right)$ is a sectionwise stable weak equivalence of presheaves of $S^{1}$-spectra.

Proof. First, the adjunction unit $\mathbb{G} \stackrel{\text { adj }}{\longrightarrow}\left(\left.\mathbb{G}_{m}^{\wedge 1}\right|_{S m / k}\right)$ in $\operatorname{sPre} \bullet(S m / k)$ induces an isomorphism $\underline{\operatorname{Hom}}\left(\mathbb{G}_{m}^{\wedge 1}, L M_{f r}\left(X \times \mathbb{G}_{m}^{\wedge 1}\right)\right) \stackrel{\text { adj }^{*}}{\longrightarrow} \underline{\operatorname{Hom}}\left(\mathbb{G}, L M_{f r}\left(X \times \mathbb{G}_{m}^{\wedge 1}\right)\right)$ of $S^{1}$-spectra. Second, the morphism $\operatorname{adj}^{*} \circ\left(-\otimes \mathbb{G}_{m}^{\wedge 1}\right)$ coincides with the morphism

$$
c_{0}: L M_{f r}(X) \rightarrow \underline{\operatorname{Hom}}\left(\mathbb{G}, L M_{f r}\left(X \times \mathbb{G}_{m}^{\wedge 1}\right)\right) .
$$

is the morphism (3). The theorem now follows from Corollary A.4.

\section{APPENDIX B. SOME FACTS ON HENZELIZATION}

We refer the reader to [Gab] or [FP] for the definition of henzelization of an affine scheme along a closed subscheme.

Let $X, X_{1}$ be $k$-smooth affine varieties, $Z \subset X, Z_{1} \subset X_{1}$ be closed subsets. Let $f: X_{1} \rightarrow X$ be a $k$-morphism such that $Z_{1} \subset f^{-1}(Z)$. For an étale neighborhood $(W, \pi: W \rightarrow X, s: Z \rightarrow W)$ of $Z$ in $X$ set $W_{1}=X_{1} \times_{X} W$. Let $\pi_{1}: W_{1} \rightarrow X_{1}$ be the projection and let $s_{1}=\left(i_{1},\left.f\right|_{Z_{1}}\right): Z_{1} \rightarrow W_{1}$, where $i_{1}: Z_{1} \hookrightarrow X_{1}$ be the inclusion. Then $\left(W_{1}, \pi_{1}, s_{1}\right)$ is an étale neighborhood of $Z_{1}$ in $X_{1}$. Denote by $f_{W}: W_{1} \rightarrow W$ the projection. Then one has a morphism $\lim \left(f_{W}\right): \lim _{(W, \pi, s)} W_{1} \rightarrow \lim _{(W, \pi, s)} W=X_{Z}^{h}$. Set,

$$
f^{h}=\lim \left(f_{W}\right) \circ \operatorname{can}_{f}:\left(X_{1}\right)_{Z_{1}}^{h} \rightarrow X_{Z}^{h},
$$


where $\operatorname{can}_{f}:\left(X_{1}\right)_{Z_{1}}^{h} \rightarrow \lim _{(W, \pi, s)} W_{1}$ is the canonical morphism. Clearly, $\rho \circ f^{h}=f \circ \rho_{1}$, where $\rho: X_{Z}^{h} \rightarrow X$ and $\rho_{1}:\left(X_{1}\right)_{Z_{1}}^{h} \rightarrow X_{1}$ are the canonical morphisms.

The following properties of the morphism $f^{h}$ are straightforward:

(1) For any affine $k$-smooth variety $X$ one has $\operatorname{id}_{X}^{h}=\mathrm{id}_{X_{Z}^{h}}$. If $p: X \rightarrow \mathrm{pt}$ is the structure map, then for any closed $Z$ in $X$ the morphism $p^{h}: X_{Z}^{h} \rightarrow(\mathrm{pt})_{\mathrm{pt}}^{h}=\mathrm{pt}$ is the structure morphism.

(2) Given a $k$-morphism $f_{1}: X_{2} \rightarrow X_{1}$ of affine $k$-smooth varieties and a closed subset $Z_{2} \subset X_{2}$ with $Z_{2} \subset f_{1}^{-1}\left(Z_{1}\right)$ one has $\left(f \circ f_{1}\right)^{h}=f^{h} \circ f_{1}^{h}$.

(3) If $i: Z \hookrightarrow X$ is the closed inclusion, $Z_{1}=Z$, then $Z_{Z}^{h}=Z$ and $i^{h}: Z=Z_{Z}^{h} \rightarrow X_{Z}^{h}$ coincides with the canonical closed inclusion $s: Z \rightarrow X_{Z}^{h}$.

The last two properties imply the following property. Let $X$ be an affine $k$-smooth variety and $x \in X$ be a $k$-rational point. Suppose $s: \mathrm{pt} \rightarrow X_{x}^{h}$ is the closed point of $X_{x}^{h}$ and $i_{x}: \mathrm{pt} \rightarrow X$ is the point $x$. Let $p: X \rightarrow \mathrm{pt}$ be the structure map. Then one has equalities

$$
\left(i_{x} \circ p\right)^{h}=i_{x}^{h} \circ p^{h}=s \circ p^{h}: X_{x}^{h} \rightarrow X_{x}^{h} .
$$

These observations imply the following

Lemma B.1. Let $X$ be an affine $k$-smooth variety and $x \in X$ be its $k$-rational point. Let $f_{s}: \mathbb{A}^{1} \times X \rightarrow$ $X$ be a morphism such that $f_{1}: X \rightarrow X$ is the identity, $f_{0}: X \rightarrow X$ coincides with the morphism $X \stackrel{p}{\rightarrow} \mathrm{pt} \stackrel{i_{x}}{\rightarrow} X$ and $f_{s}\left(\mathbb{A}^{1} \times\{x\}\right)=\{x\}$. Then the morphism $f_{s}^{h}:\left(\mathbb{A}^{1} \times X\right)_{\mathbb{A}^{1} \times x}^{h} \rightarrow X_{x}^{h}$ defined by (8) has the following properties:

(1) $\left.\left(f_{s}^{h}\right)\right|_{(1 \times X)_{(1, x)}^{h}}: X_{x}^{h} \rightarrow X_{x}^{h}$ is the identity;

(2) $\left.\left(f_{s}^{h}\right)\right|_{(0 \times X)_{(0, x)}^{h}}: X_{x}^{h} \rightarrow X_{x}^{h}$ coincides with the morphism $X_{x}^{h} \stackrel{p^{h}}{\rightarrow} \mathrm{pt} \stackrel{s_{x}}{\rightarrow} X_{x}^{h}$, where $s_{x}: \mathrm{pt} \hookrightarrow X_{x}^{h}$ is the closed point of $X_{x}^{h}$.

Proof. The first assertion follows from the equalities

$$
\operatorname{id}_{X_{Z}^{h}}=\operatorname{id}_{X}^{h}=\left(f_{1}\right)^{h}=\left(f_{s} \circ i_{1}\right)^{h}=f_{s}^{h} \circ i_{1}^{h}=\left.\left(f_{s}^{h}\right)\right|_{(1 \times X)_{(1, x)}^{h}} .
$$

Let $s_{x}: \mathrm{pt} \hookrightarrow X_{x}^{h}$ be the closed point of $X_{x}^{h}$. As mentioned above, $s_{x}=i_{x}^{h}$, where $i_{x}: \mathrm{pt} \rightarrow X$ is the closed point $x$ of $X$. The equalities

$$
s_{x} \circ p^{h}=i_{x}^{h} \circ p^{h}=\left(i_{x} \circ p\right)^{h}=f_{0}^{h}=\left(f_{s} \circ i_{0}\right)^{h}=f_{s}^{h} \circ i_{0}^{h}=\left.\left(f_{s}^{h}\right)\right|_{(0 \times X)_{(0, x)}^{h}}
$$

imply the second assertion.

If we take $X=\mathbb{A}^{m}$, a $k$-rational point $x \in \mathbb{A}^{m}$ and the morphism $f_{s}: \mathbb{A}^{1} \times \mathbb{A}^{m} \rightarrow \mathbb{A}^{m}$ mapping $(s, y)$ to $s \cdot(y-x)+x$, then $f_{s}: \mathbb{A}^{1} \times X \rightarrow X$ satisfies the hypotheses of Lemma B.1. Thus Lemma B.1 implies the following statement, which is in fact Lemma 4.10.

Corollary B.2. The morphism $H_{s}:=f_{s}^{h}: U_{s}^{\prime} \rightarrow U^{\prime}$ has the following properties:

(a) $H_{1}:=\left.\left(f_{s}^{h}\right)\right|_{(1 \times X)_{(1, x)}^{h}}: U^{\prime} \rightarrow U^{\prime}$ is the identity morphism;

(b) $H_{0}:=\left.\left(f_{s}^{h}\right)\right|_{(0 \times X)_{(0, x)}^{h}}: U^{\prime} \rightarrow U^{\prime}$ coincides with the composite morphism $U^{\prime} \stackrel{p^{h}}{\rightarrow} p \stackrel{s_{x}}{\rightarrow} U^{\prime}$, where $p^{h}: U^{\prime} \rightarrow p t=\operatorname{Spec}(k)$ is the structure morphism and $s_{x}: \mathrm{pt} \hookrightarrow X_{x}^{h}$ is the closed point of $X_{x}^{h}$. 


\section{ACKNOWLEDGEMENTS}

This paper was partly written during the visit of the authors in summer 2014 to the University of Duisburg-Essen (Marc Levine's Arbeitsgruppe). They would like to thank the University for its kind hospitality and support. The first author and the third author were supported by a grant from the Government of the Russian Federation (agreement 075-15-2019-1620). The first author was also supported by the Young Russian Mathematics award.

\section{REFERENCES}

[DP] A. Druzhinin, I. Panin, Surjectivity of the etale excision map for homotopy invariant framed presheaves, preprint arXiv: 1808.07765 .

[FP] R. Fedorov, I. Panin, A proof of the Grothendieck-Serre conjecture on principal bundles over regular local rings containing infinite fields, Publ. Math. IHES 122(1) (2015), 169-193.

[Gab] O. Gabber, Affine analog of the proper base change theorem, Israel J. Math., 87 (1994), 325-335.

[GNP] G. Garkusha, A. Neshitov, I. Panin, Framed motives of relative motivic spheres, Trans. Amer. Math. Soc., to appear. DOI https://doi.org/10.1090/tran/8386.

[GP1] G. Garkusha, I. Panin, Framed motives of algebraic varieties (after V. Voevodsky), J. Amer. Math. Soc. 34(1) (2021), 261-313.

[GP2] G. Garkusha, I. Panin, Homotopy invariant presheaves with framed transfers, Cambridge J. Math. 8(1) (2020), $1-94$.

[GP3] G. Garkusha, I. Panin, The triangulated categories of framed bispectra and framed motives, preprint arXiv: 1809.08006.

[GP4] G. Garkusha, I. Panin, On the motivic spectral sequence, J. Inst. Math. Jussieu 17(1) (2018), 137-170.

[EGA4] A. Grothendieck, Éléments de géométrie algébrique. IV, Étude locale des schemas et des morphismes de schemas, Quatrième partie, Publ. Math. IHES 32 (1967), 5-361.

[GrD] A. Grothendieck, J. Dieudonné, Éléments de Géométrie Algébrique IV. Étude locale des schémas et des morphismes de schémas (Troisième Partie), Publ. Math. IHÉS 28 (1966), 5-255.

[Jar] J. F. Jardine, Motivic symmetric spectra, Doc. Math. 5 (2000), 445-552.

[Hir] Ph. S. Hirschhorn, Model categories and their localizations, Mathematical Surveys and Monographs 99, 2003.

[MV] F. Morel, V. Voevodsky, $\mathbb{A}^{1}$-homotopy theory of schemes, Publ. Math. IHES 90 (1999), 45-143.

[OP] M.Ojanguren, I.Panin, Rationally trivial hermitian spaces are locally trivial, Math. Z. 237(1) (2001), 181-198.

[Sch] S. Schwede, An untitled book project about symmetric spectra, available at www.math.unibonn.de/people/schwede/SymSpec-v3.pdf (version April 2012).

[Seg] G. Segal, Categories and cohomology theories, Topology 13 (1974), 293-312.

[S] A. Suslin, On the Grayson spectral sequence, Proc. Steklov Inst. Math. 241 (2003), 202-237.

[V1] V. Voevodsky, Notes on framed correspondences, unpublished, 2001. Also available at https://www.math.ias.edu/vladimir/publications

[V2] V. Voevodsky, Cancellation theorem, Doc. Math. Extra Volume in honor of A. Suslin (2010), 671-685.

St. Petersburg Branch of Steklov Mathematical Institute, Fontanka 27, St. Petersburg, 191023, RUSSIA

Email address: alseang@gmail.com

Department of Mathematics, Swansea University, Fabian Way, SWAnsea SA1 8EN, United KingDOM

Email address: g.garkusha@swansea.ac.uk

St. Petersburg Branch of Steklov Mathematical Institute, Fontanka 27, St. Petersburg, 191023, RUSSIA

Email address: paniniv@gmail.com 